\title{
Piatetski-Shapiro meets Chebotarev
}

\author{
by \\ Yildirim Akbal and Ahmet Muhtar GüloĞLu (Ankara)
}

1. Introduction. In 1953 Ilya Piatetski-Shapiro 12 proved an analog of the prime number theorem for primes of the form $\left\lfloor n^{c}\right\rfloor$ where $\lfloor x\rfloor=$ $\max \{n \in \mathbb{N}: n \leq x\}, n$ runs through positive integers and $c>0$ is fixed. He showed that such primes constitute a thin subset of the primes; more precisely, the number $\pi_{c}(x)$ of these primes not exceeding a given number $x$ is asymptotic to $x^{1 / c} / \log x$, provided that $c \in(1,12 / 11)$. Since then, the admissible range of $c$ has been extended by many authors and the result is currently known for $c \in(1,2817 / 2426)$ (cf. [13]).

A related question is to determine the asymptotic behavior of a particular subset of these primes, for example, those belonging to a given arithmetic progression, or those of the form $a^{2}+n b^{2}$. The former was considered by Leitmann and Wolke [8] in 1974, and it has been used in a recent paper by Baker et al. [1] to show the existence of infinitely many Carmichael numbers that are products of Piateski-Shapiro primes.

For both of the aforementioned examples, the problem can be interpreted as counting the Piatetski-Shapiro primes that belong to a particular Chebotarev class of some number field (see Theorem 1 and the remark following Theorem 2). Motivated by this observation, we study the following more general problem:

Take a finite Galois extension $K / \mathbb{Q}$ and a conjugacy class $C$ in the Galois group $G=\operatorname{Gal}(K / \mathbb{Q})$. Set

$$
\pi(K, C)=\left\{p \text { prime }: \operatorname{gcd}\left(p, \Delta_{K}\right)=1,[K / \mathbb{Q}, p]=C\right\}
$$

where $\Delta_{K}$ is the discriminant of $K$, and the Artin symbol $[K / \mathbb{Q}, p]$ is defined as the conjugacy class of the Frobenius automorphism associated with any prime ideal $\mathfrak{P}$ of $K$ above $p$. Recall that the Frobenius automorphism is the

2010 Mathematics Subject Classification: Primary 11L07; Secondary 11R45, 11B83.

Key words and phrases: Chebotarev's density theorem, Piatetski-Shapiro prime number theorem, exponential sums over ideals, generalized Vaughan's identity, van der Corput's method, Vinogradov's method. 
generator of the decomposition group of $\mathfrak{P}$, which is the cyclic subgroup of automorphisms of $G$ that fixes $\mathfrak{P}$. The Chebotarev density theorem as given by Lemma 5 below states that the natural density of primes in $\pi(K, C)$ is $|C| /|G|$; that is,

$$
\pi(K, C, x) \sim \frac{|C|}{|G|} \operatorname{li}(x) \quad(x \rightarrow \infty)
$$

where $\pi(K, C, x)=\#\{p \leq x: p \in \pi(K, C)\}$ and $\operatorname{li}(x)=\int_{2}^{x}(\log t)^{-1} d t$ is the logarithmic integral.

Our intent in this paper is to find an asymptotic formula for the number of Piatetski-Shapiro primes that belong to $\pi(K, C)$. To this end, we define the counting function

$$
\pi_{c}(K, C, x)=\#\left\{p \leq x: p \in \pi(K, C), p=\left\lfloor n^{c}\right\rfloor \text { for some } n \in \mathbb{N}\right\} .
$$

The first result we prove in this direction is for abelian extensions $K / \mathbb{Q}$. By the Kronecker-Weber theorem this problem easily reduces to counting the Piatetski-Shapiro primes in an arithmetic progression, which was handled in [8] as we have mentioned above. We do, however, reprove their theorem here in a slightly different manner following a more recent method given in [4, §4.6] that utilizes Vaughan's identity.

Before stating our first result, we recall that the conductor $\mathfrak{f}$ of an abelian extension $K / \mathbb{Q}$ is the modulus of the smallest ray class field $K^{\mathfrak{f}}$ containing $K$.

TheOREm 1. Let $K / \mathbb{Q}$ be an abelian extension of conductor $\mathfrak{f}$. Take any automorphism $\sigma$ in the Galois group $G=\operatorname{Gal}(K / \mathbb{Q})$. Then there exists an absolute constant $D>0$ and a constant $x_{0}(\mathfrak{f})$ such that for any fixed $c \in(1,12 / 11)$ and $x \geq x_{0}(\mathfrak{f})$ we have

$$
\pi_{c}(K,\{\sigma\}, x)=\frac{1}{c|G|} \operatorname{li}\left(x^{1 / c}\right)+O\left(x^{1 / c} \exp (-D \sqrt{\log x})\right)
$$

where the implied constant depends only on $c$.

Next, we consider a non-abelian Galois extension $K / \mathbb{Q}$. Given a conjugacy class $C$ in $G$, take any representative $\sigma \in C$ and set $d_{L}=[G:\langle\sigma\rangle]=$ $[L: \mathbb{Q}]$ where $L$ is the fixed field corresponding to the cyclic subgroup $\langle\sigma\rangle$ of $G$ generated by $\sigma$. Note that $d_{L} \geq 2$. As in the abelian case, we obtain a similar asymptotic formula, only this time the range of $c$ depends on the size of $d_{L}$ (not on $L$, hence $\sigma$ ). This is due to the nature of an exponential sum that appears in the estimate of one of the error terms. In this case, we prove the following result:

Theorem 2. Let $K, C, G$ and $d_{L}$ be as defined above. Then there exists an absolute constant $D>0$ and a constant $x_{0}$ which depends on the degree $d_{K}$ and the discriminant $\Delta_{K}$ of $K$ such that, for $x \geq x_{0}$ and for $c$ 
that satisfies

$$
1<c<1+ \begin{cases}\left(2^{d_{L}+1} d_{L}+1\right)^{-1} & \text { if } d_{L} \leq 10 \\ \left(6\left(d_{L}^{3}+d_{L}^{2}\right) \log \left(125 d_{L}\right)-1\right)^{-1} & \text { otherwise }\end{cases}
$$

we have

$$
\pi_{c}(K, C, x)=\frac{|C|}{c|G|} \operatorname{li}\left(x^{1 / c}\right)+O\left(x^{1 / c} \exp \left(-D\left|\Delta_{K}\right|^{-1 / 2}(\log x)^{1 / 2}\right)\right)
$$

where the implied constant depends on $c$, the degree $d_{L}$ and the discriminant $\Delta_{L}$ of the intermediate field $L$ defined above.

The asymptotic formula above follows from the effective version of the Chebotarev density theorem (see Lemma 5) coupled with an adaptation of the method in [4, §4.6] to our case using an analog of Vaughan's identity for number fields (see Lemma 10). The main difference from [4, §4.6] is that here one has to deal with the estimate of an exponential sum that runs over the integral ideals of $L$ (see $\$ 2.3,82.7$ ), and most of the paper is devoted to estimating this sum. In a nutshell, to handle the twisted exponential sum in $\S 2.3$ we first split it into ray classes (removing the character). Choosing an integral basis then for each resulting sum, we are eventually led to the multi-dimensional exponential sum in (2.8). At this point, we estimate the innermost sum by van der Corput's method for small values of $d_{L}$, and Vinogradov's method for larger $d_{L}$, and the rest of the sums are estimated trivially.

As an application, we consider the ring class field $L_{n}$ of the order $\mathbb{Z}[\sqrt{-n}]$ in the imaginary quadratic field $K=\mathbb{Q}(\sqrt{-n})$ where $n$ is a positive integer. It follows from [3, Lemma 9.3] that $L_{n}$ is a Galois extension of $\mathbb{Q}$ with Galois group isomorphic to $\operatorname{Gal}\left(L_{n} / K\right) \rtimes(\mathbb{Z} / 2 \mathbb{Z})$ where the non-trivial element of $\mathbb{Z} / 2 \mathbb{Z}$ acts on $\operatorname{Gal}\left(L_{n} / K\right)$ by sending $\sigma$ to its inverse $\sigma^{-1}$. For example, $\operatorname{Gal}\left(L_{27} / \mathbb{Q}\right) \simeq S_{3}$ is non-abelian, while $\operatorname{Gal}\left(L_{3} / \mathbb{Q}\right)$ is abelian since $L_{3}=$ $\mathbb{Q}(\sqrt{-3})$. In any case, we know from [3, Theorem 9.4] that if $p$ is an odd prime not dividing $n$ then $p=a^{2}+n b^{2}$ for some integers $a, b$ if and only if $p$ splits completely in $L_{n}$, which occurs exactly when $\left[L_{n} / \mathbb{Q}, p\right]$ is the identity automorphism $\mathbf{1}_{G}$ of $G=\operatorname{Gal}\left(L_{n} / \mathbb{Q}\right)$. Therefore, as a corollary of the theorems above we see that the number of Piatetski-Shapiro primes up to $x$ that are of the form $a^{2}+n b^{2}$ is asymptotic to $(c|G|)^{-1} \operatorname{li}\left(x^{1 / c}\right)$ as $x \rightarrow \infty$ for any $c$ in the range given by the relevant theorem above depending on whether $L_{n} / \mathbb{Q}$ is abelian. Note that by [3, Lemma 9.3], $L_{n} / \mathbb{Q}$ is abelian only if $\left[L_{n}: K\right] \leq 2$. On the other hand, $\left[L_{n}: K\right]$ is the class number $h(\mathbb{Z}[\sqrt{-n}])$ of the order $\mathbb{Z}[\sqrt{-n}]$, which by [3, Theorem 7.24] is an integral multiple of $h(K)$. Since it is also known that there are only finitely many $n$ such that $h(K) \leq 2$, we conclude that for all but finitely many $n>0, L_{n} / \mathbb{Q}$ is non-abelian. 
REMARK 3. Adapting the most recent methods that have been used for the classical Piatetski-Shapiro problem it may be possible to obtain a slightly larger range for $c$ in both Theorems 1 and 2, although we have not attempted to do so for the sake of simplicity.

REMARK 4. If one assumes GRH for the Dedekind zeta function of $K$, then the best one can show with our methods is that the asymptotic formula

$$
\pi_{c}(K, C, x)=\frac{|C|}{c|G|} \operatorname{li}\left(x^{1 / c}\right)+O\left(x^{1 / c-\epsilon(c)}\right)
$$

holds for sufficiently large $x$ and with an $\epsilon(c)>0$ that approaches zero as $c$ tends to the upper limit of its range given in Theorems 1 and 2. Note that it is also possible to give an explicit expression for $\epsilon(c)$, but this requires some extra work. One can also get an error of the form $O\left(x^{1 / c-\epsilon}\right)$ for a fixed small $\epsilon>0$ at the expense of a smaller range for $c$.

1.1. Preliminaries and notation. We use Vinogradov's notation $f \ll g$ to mean that $|f(x)| \leq C g(x)$, where $g$ is a positive function and $C>0$ is a constant. Similarly, we define $f \gg g$ to mean $|f| \geq C g$ and $f \asymp g$ to mean both $f \ll g$ and $f \gg g$.

We write $e(z)$ for $\exp (2 \pi i z)$.

For any finite field extension $L / \mathbb{Q}$, we write $\Delta_{L}$ for its absolute discriminant and $d_{L}$ for its degree $[L: \mathbb{Q}]=r_{1}+2 r_{2}$ where $r_{1}$ is the number of real embeddings of $L$. We denote the ring of integers of $L$ by $\mathfrak{O}_{L}$, and the absolute norm of an ideal $\mathfrak{a}$ is denoted by $\mathfrak{N a}$.

The letter $p$ always denotes an ordinary prime number. Similarly, we use the letters $\mathfrak{p}, \mathfrak{P}$ for prime ideals.

Preliminaries. Here we state some auxiliary lemmas that will be needed for the proof of Theorem 2 .

LEMma 5 (Chebotarev density theorem). Let $K / \mathbb{Q}$ be a Galois extension and $C$ a conjugacy class in the Galois group $G$. If $d_{K}>1$, there exists an absolute, effectively computable constant $D$ and a constant $x_{0}=x_{0}\left(d_{K}, \Delta_{K}\right)$ such that if $x \geq x_{0}$, then

$$
\pi(K, C, x)=\frac{|C|}{|G|} \operatorname{li}(x)+O\left(x \exp \left(-D\left|\Delta_{K}\right|^{-1 / 2} \sqrt{\log x}\right)\right)
$$

where the implied constant is absolute. Furthermore, if GRH holds for the Dedekind zeta function of $K$, then for $x \geq 2$,

$$
\left|\pi(K, C, x)-\frac{|C|}{|G|} \operatorname{li}(x)\right| \leq c_{1}\left(\frac{|C|}{|G|} x^{1 / 2} \log \left(\left|\Delta_{K}\right| x^{d_{K}}\right)+\log \left|\Delta_{K}\right|\right)
$$

where $c_{1}$ is an effectively computable positive absolute constant. 
Proof. The result immediately follows by combining [7, Theorems 1.1, 1.3 and 1.4].

We refer to [2, Lemma 2] for the following result.

Lemma 6. Let $L / \mathbb{Q}$ be a finite extension of degree $d_{L}$ and discriminant $\Delta_{L}$. For each ideal $\mathfrak{a}$ of $L$, there exists a basis $\alpha_{1}, \ldots, \alpha_{d_{L}}$ such that for any embedding $\tau$ of $L$,

$$
A_{1}^{-d_{L}+1}(\mathfrak{N a})^{1 /\left(2 d_{L}\right)} \leq\left|\tau \alpha_{j}\right| \leq A_{1}(\mathfrak{N a})^{1 / d_{L}}
$$

where $A_{1}=d_{L}^{d_{L}}\left|\Delta_{L}\right|^{1 / 2}$.

For the proof of the next lemma, see for example [6, Theorem 11.8].

Lemma 7. Let $L$ be a finite extension and $\mathfrak{U}$ be a non-zero ideal in the ring of integers $\mathfrak{O}_{L}$. There exists an element $\alpha \neq 0$ in $\mathfrak{U}$ such that

$$
\mathfrak{N}\left(\alpha \mathfrak{U}^{-1}\right) \leq \frac{d_{L} !}{d_{L}^{d_{L}}}\left(\frac{4}{\pi}\right)^{r_{2}}\left|\Delta_{L}\right|^{1 / 2}
$$

where $2 r_{2}$ is the number of complex embeddings of $L$.

2. Proof of Theorem 2, We start with the observation that the expression $\left\lfloor-p^{\delta}\right\rfloor-\left\lfloor-(p+1)^{\delta}\right\rfloor$ is either 0 or 1 , where $\delta=1 / c$, and the latter holds exactly when $p=\left\lfloor n^{c}\right\rfloor$ for some $n \in \mathbb{N}$. Using this characterization and the identity

$$
\begin{aligned}
\left\lfloor-p^{\delta}\right\rfloor-\left\lfloor-(p+1)^{\delta}\right\rfloor & =(p+1)^{\delta}-p^{\delta}+\psi\left(-(p+1)^{\delta}\right)-\psi\left(-p^{\delta}\right) \\
& =\delta p^{\delta-1}+O\left(p^{\delta-2}\right)+\psi\left(-(p+1)^{\delta}\right)-\psi\left(-p^{\delta}\right)
\end{aligned}
$$

where $\psi(x)=x-\lfloor x\rfloor-1 / 2$, we obtain

$$
\pi_{c}(K, C, x)=\sum_{\substack{p \leq x \\ p \in \pi(K, C)}} \delta p^{\delta-1}+\sum_{\substack{p \leq x \\ p \in \pi(K, C)}}\left(\psi\left(-(p+1)^{\delta}\right)-\psi\left(-p^{\delta}\right)\right)+O(\log x) .
$$

By partial summation, it follows from Lemma 5 that for $x \geq x_{0}=$ $x_{0}\left(d_{K},\left|\Delta_{K}\right|\right)$,

$$
\sum_{\substack{p \leq x \\ p \in \pi(K, C)}} \delta p^{\delta-1}=\frac{|C|}{c|G|} \operatorname{li}\left(x^{1 / c}\right)+O\left(x^{1 / c} \exp \left(-D\left|\Delta_{K}\right|^{-1 / 2} \sqrt{\log x}\right)\right)
$$

where the implied constant is absolute.

The rest of this section deals with the estimate of the sum involving $\psi$. For any function $f(x)$, we put $F(f, x)=f\left(-(x+1)^{\delta}\right)-f\left(-x^{\delta}\right)$. Using dyadic division yields

$$
\sum_{\substack{p \leq x \\ p \in \pi(K, C)}} F(\psi, p)=\sum_{\substack{1 \leq N<x \\ N=2^{k}}} \sum_{\substack{N<p<N_{1} \\ p \in \pi(K, C)}} F(\psi, p)
$$


where $N_{1}=\min (x, 2 N)$. By Vaaler's theorem (see, e.g., [4, Appendix]) we can approximate $\psi(x)$ with the function

$$
\psi^{*}(x)=\sum_{1 \leq|h| \leq H} a_{h} e(h x) \quad\left(a_{h} \ll h^{-1}\right)
$$

where the error estimate $\psi(x)-\psi^{*}(x) \ll \Delta(x)$ holds for some non-negative function $\Delta$ given by

$$
\Delta(x)=\sum_{|h|<H} b(h) e(h x) \quad\left(b(h) \ll H^{-1}\right) .
$$

Using the definition of $\Delta$, we deduce from [4, p. 48] that

$$
\sum_{\substack{N<p \leq N_{1} \\ p \in \pi(K, C)}} F\left(\psi-\psi^{*}, p\right) \ll \sum_{N<n \leq N_{1}} \Delta\left(-n^{\delta}\right) \ll N H^{-1}+N^{\delta / 2} H^{1 / 2} .
$$

Thus, taking

$$
H=N^{1-\delta+\varepsilon}
$$

yields

$$
\sum_{p \in \pi(K, C, x)} F\left(\psi-\psi^{*}, p\right) \ll x^{\delta} \exp \left(-D\left|\Delta_{K}\right|^{-1 / 2} \sqrt{\log x}\right)
$$

provided that $1<c<2$ and $\varepsilon>0$ is sufficiently small, both of which are assumed in what follows.

Having dealt with the error term, we now turn to the sum involving $\psi^{*}$. Using partial summation we obtain

$$
\sum_{\substack{N<p \leq N_{1} \\ p \in \pi(\bar{K}, C)}} F\left(\psi^{*}, p\right) \ll \frac{1}{\log N} \max _{N^{\prime} \in\left(N, N_{1}\right]}\left|\sum_{\substack{N<n \leq N^{\prime} \\ n \in\langle\pi(\bar{K}, C)\rangle}} F\left(\psi^{*}, n\right) \Lambda(n)\right|+O(\sqrt{N})
$$

where $\langle\pi(K, C)\rangle$ denotes the set of integers whose prime factors belong to $\pi(K, C)$. Recalling the definition of $\psi^{*}$ above we derive that

$$
\begin{aligned}
\sum_{\substack{N<n<N^{\prime} \\
n \in\langle\pi(K, C)\rangle}} F\left(\psi^{*}, n\right) \Lambda(n) & =\sum_{1 \leq|h| \leq H} a_{h} \sum_{\substack{N<n \leq N^{\prime} \\
n \in\langle\pi(K, C)\rangle}} F(e(h x), n) \Lambda(n) \\
& \ll \sum_{1 \leq h \leq H} h^{-1 \mid} \sum_{\substack{N<n \leq N^{\prime} \\
n \in\langle\pi(K, C)\rangle}} e\left(h n^{\delta}\right) \phi_{h}(n) \Lambda(n) \mid
\end{aligned}
$$

where $\phi_{h}(x)=1-e\left(h\left((x+1)^{\delta}-x^{\delta}\right)\right)$. Using the bounds

$$
\phi_{h}(x) \ll h x^{\delta-1}, \quad \phi_{h}^{\prime}(x) \ll h x^{\delta-2}
$$


and partial summation we see that the inner sum above is

$$
\ll h N^{\delta-1} \max _{N^{\prime} \in\left(N, N_{1}\right]}\left|\sum_{\substack{N<n \leq N^{\prime} \\ n \in\langle\pi(K, C)\rangle}} e\left(h n^{\delta}\right) \Lambda(n)\right| .
$$

Thus, to finish the proof of Theorem 2 it is enough to show that

$$
\sum_{h}\left|\sum_{\substack{N<n \leq N^{\prime} \\ n \in\langle\pi(K, C)\rangle}} e\left(h n^{\delta}\right) \Lambda(n)\right| \ll N \exp \left(-D\left|\Delta_{K}\right|^{-1 / 2} \sqrt{\log N}\right) .
$$

Lemma 8. Take a representative $\sigma \in C$. Let $L$ be the fixed field of the cyclic group $\langle\sigma\rangle$ generated by $\sigma$. Then, for $N^{\prime} \leq N_{1} \leq 2 N$,

$$
\begin{aligned}
& \sum_{\substack{N<n<N^{\prime} \\
n \in\langle\pi(K, C)\rangle}} e\left(h n^{\delta}\right) \Lambda(n) \\
& \quad=\frac{|C|}{|G|} \sum_{\psi} \overline{\psi(\sigma)} \sum_{\substack{\mathfrak{a} \subseteq \mathfrak{O}_{L} \\
N<\overline{\mathfrak{N}} \leq N^{\prime}}} \psi([K / L, \mathfrak{a}]) \Lambda_{L}(\mathfrak{a}) e\left(h(\mathfrak{N a})^{\delta}\right)+O(\sqrt{N})
\end{aligned}
$$

where the first summation is taken over all characters of $\operatorname{Gal}(K / L)$ and the second is over powers of prime ideals of $L$ that are unramified in $K$.

Proof. Since $K / L$ is abelian, by the orthogonality of characters of $\operatorname{Gal}(K / L)$ the expression

$$
\sum_{\psi} \overline{\psi(\sigma)} \sum_{\substack{\mathfrak{a} \subseteq \mathfrak{O}_{L} \\ N<\mathfrak{N} \mathfrak{a} \leq N^{\prime}}} \psi([K / L, \mathfrak{a}]) \Lambda_{L}(\mathfrak{a}) e\left(h(\mathfrak{N a})^{\delta}\right)
$$

equals

$$
\operatorname{ord}_{G}(\sigma) \sum_{\substack{\mathfrak{a} \subseteq \mathfrak{O}_{L} \\ N<\overline{\mathfrak{N a}} \leq N^{\prime} \\[K / L, \mathfrak{a}]=\sigma}} \Lambda_{L}(\mathfrak{a}) e\left(h(\mathfrak{N a \mathfrak { a }})^{\delta}\right)
$$

By removing prime ideals $\mathfrak{p}$ of $L$ with $\operatorname{deg} \mathfrak{p}>1$ and powers of prime ideals $\mathfrak{p}^{k}$ with $k>1$, the last sum can be written as

$$
\sum_{\substack{N<\mathfrak{N p} \leq N^{\prime} \\[K / L, \mathfrak{p}]=\sigma \\ \mathfrak{N} p \text { is prime }}} e\left(h(\mathfrak{N p})^{\delta}\right) \log \mathfrak{N p}+O(\sqrt{N})
$$

or

$$
\sum_{N<p \leq N^{\prime}}\left(\sum_{\substack{\mathfrak{p} \subseteq \mathfrak{O}_{L} \\[K / L, \mathfrak{p}]=\sigma \\ \mathfrak{N} \mathfrak{p}=p}} 1\right) e\left(h p^{\delta}\right) \log p+O(\sqrt{N})
$$


If $p$ is a prime that is unramified in $K$, and $\mathfrak{p}$ is a prime ideal of $L$ above $p$ satisfying $[K / L, \mathfrak{p}]=\sigma$, then $\mathfrak{p}$ remains prime in $K$ and

$$
[K / L, \mathfrak{p}]=\sigma \text { and } \mathfrak{N p}=p \Leftrightarrow\left[K / \mathbb{Q}, \mathfrak{p} \mathfrak{O}_{K}\right]=\sigma .
$$

In particular, $[K / \mathbb{Q}, p]=C$. Furthermore, the number of prime ideals $\mathfrak{P}$ of $K$ above such a prime $p$ with $[K / \mathbb{Q}, \mathfrak{P}]=\sigma$ equals $\left[C_{G}(\sigma):\langle\sigma\rangle\right]$ where $C_{G}(\sigma)$ is the centralizer of $\sigma$ in $G$. The result now follows by observing that $\left|C_{G}(\sigma)\right|=|G| /|C|$ and noting that

$$
\sum_{\substack{N<n \leq N^{\prime} \\ n \in\langle\pi(K, C)\rangle}} e\left(h n^{\delta}\right) \Lambda(n)=\sum_{\substack{p \in \pi(K, C) \\ N<p \leq N^{\prime}}} e\left(h p^{\delta}\right) \log p+O(\sqrt{N}) .
$$

REMARK 9. From now on we shall write $\chi(\mathfrak{a})$ for the composition $\Psi([K / L, \mathfrak{a}])$. Note that since $K / L$ is abelian, $\chi$ is a character of the ray class group $J^{\mathfrak{f}} / P^{\mathfrak{f}}$ (see, e.g., [10, p. 525]) where $\mathfrak{f}$ is the conductor of the extension $K / L$. Furthermore, we shall require that $\chi(\mathfrak{a})=0$ whenever $\mathfrak{a}$ is not coprime to $\mathfrak{f}$. This way, we can assume that the inner sum in the lemma above runs over all integral ideals of $L$.

Our current objective is to prove that

$$
\sum_{h}\left|\sum_{\substack{\mathfrak{a} \subseteq \mathfrak{O}_{L} \\ N<\mathfrak{N a} \leq N^{\prime}}} \chi(\mathfrak{a}) \Lambda_{L}(\mathfrak{a}) e\left(h(\mathfrak{N a})^{\delta}\right)\right| \ll N \exp \left(-D\left|\Delta_{K}\right|^{-1 / 2} \sqrt{\log N}\right) .
$$

We start with an analog of Vaughan's identity for number fields.

Lemma 10. Let $u, v \geq 1$. For any ideal $\mathfrak{a} \subseteq \mathfrak{O}_{L}$ with $\mathfrak{N a}>u$,

$$
\begin{aligned}
\Lambda_{L}(\mathfrak{a})= & \sum_{\substack{\mathfrak{b}=\mathfrak{a} \\
\mathfrak{T} \mathfrak{b} \leq v}} \mu_{L}(\mathfrak{b}) \log \mathfrak{N} \mathfrak{c} \\
& -\sum_{\substack{\mathfrak{b} \mathfrak{d}=\mathfrak{a} \\
\mathfrak{N} \mathfrak{b} \leq v, \mathfrak{\mathfrak { N }}_{\mathfrak{c}} \leq u}} \mu_{L}(\mathfrak{b}) \Lambda_{L}(\mathfrak{c})-\sum_{\substack{\mathfrak{c}=\mathfrak{a} \\
\mathfrak{N} \mathfrak{c}>u, \mathfrak{N} \mathfrak{e}>v}} \Lambda_{L}(\mathfrak{c}) \sum_{\substack{\mathfrak{b} \mathfrak{d}=\mathfrak{e} \\
\mathfrak{N} \mathfrak{b} \leq v}} \mu_{L}(\mathfrak{b})
\end{aligned}
$$

where

$$
\begin{aligned}
& \mu_{L}(\mathfrak{a})= \begin{cases}(-1)^{k} & \text { if } \mathfrak{a}=\mathfrak{p}_{1} \cdots \mathfrak{p}_{k}, \\
0 & \text { otherwise }\end{cases} \\
& \Lambda_{L}(\mathfrak{a})= \begin{cases}\log \mathfrak{N \mathfrak { p }} & \text { if } \mathfrak{a}=\mathfrak{p}^{k} \text { for some } k \geq 1, \\
0 & \text { otherwise }\end{cases}
\end{aligned}
$$

Proof. We use the identity

$$
\Lambda_{L}(\mathfrak{a})=\sum_{\mathfrak{b} \mathfrak{c}=\mathfrak{a}} \mu_{L}(\mathfrak{b}) \log \mathfrak{N} \mathfrak{c}
$$


and then follow the argument preceding [5, Proposition 13.4]. Finally, note that

$$
\begin{aligned}
& \sum_{\substack{\mathfrak{b} \mathfrak{d}=\mathfrak{a} \\
\mathfrak{N} \mathfrak{b}>v, \mathfrak{\mathfrak { N }}>>u}} \mu_{L}(\mathfrak{b}) \Lambda_{L}(\mathfrak{c})=\sum_{\substack{\mathfrak{c} \mathfrak{c}=\mathfrak{a} \\
\mathfrak{N} \mathfrak{c}>u}} \Lambda_{L}(\mathfrak{c}) \sum_{\substack{\mathfrak{b} \mathfrak{d}=\mathfrak{e} \\
\mathfrak{N} \mathfrak{b}>v}} \mu_{L}(\mathfrak{b}) \\
& =\sum_{\substack{\mathfrak{c}=\mathfrak{a} \\
\mathfrak{N} \mathfrak{c}>u, \mathfrak{N} \mathfrak{l}>v}} \Lambda_{L}(\mathfrak{c})\left(\sum_{\substack{\mathfrak{b} \mathfrak{d}=\mathfrak{e} \\
\mu_{L}}}(\mathfrak{b})-\sum_{\substack{\mathfrak{h} \mathfrak{d}=\mathfrak{e} \\
\mathfrak{N} \mathfrak{b} \leq v}} \mu_{L}(\mathfrak{b})\right) \\
& =-\sum_{\substack{\mathfrak{c}=\mathfrak{a} \\
\mathfrak{N}>u, \mathfrak{N} \mathfrak{e}>v}} \Lambda_{L}(\mathfrak{c}) \sum_{\substack{\mathfrak{b} \mathfrak{b}=\mathfrak{e} \\
\mathfrak{N} \mathfrak{b} \leq v}} \mu_{L}(\mathfrak{b}) .
\end{aligned}
$$

We assume from now on that $u<N$. It follows from Lemma 10 that

$$
\sum_{\substack{\mathfrak{a} \subseteq \mathfrak{O}_{L} \\ N<\mathfrak{N} \mathfrak{a} \leq N^{\prime}}} \chi(\mathfrak{a}) \Lambda_{L}(\mathfrak{a}) e\left(h(\mathfrak{N a \mathfrak { a }})^{\delta}\right)=S_{1}+S_{2}+S_{3}
$$

where

$$
\begin{aligned}
& S_{1}=-\sum_{\substack{\mathfrak{a} \subseteq \mathfrak{O}_{L} \\
N<\mathfrak{N} \mathfrak{a} \leq N^{\prime}}} \chi(\mathfrak{a}) e\left(h(\mathfrak{N a})^{\delta}\right) \sum_{\substack{\mathfrak{c}=\mathfrak{a} \\
\mathfrak{N}>u, \mathfrak{N} \mathfrak{e}>v}} \Lambda_{L}(\mathfrak{c}) \sum_{\substack{\mathfrak{b} \mathfrak{d}=\mathfrak{e} \\
\mathfrak{N} \mathfrak{b} \leq v}} \mu_{L}(\mathfrak{b}), \\
& S_{2}=\sum_{\substack{\mathfrak{a} \subseteq \mathfrak{O}_{L} \\
N<\mathfrak{N} \mathfrak{a} \leq N^{\prime}}} \chi(\mathfrak{a}) e\left(h(\mathfrak{N a})^{\delta}\right) \sum_{\substack{\mathfrak{b} \mathfrak{c}=\mathfrak{a} \\
\mathfrak{N} b \leq v}} \mu_{L}(\mathfrak{b}) \log \mathfrak{N} \mathfrak{c}, \\
& S_{3}=-\sum_{\substack{\mathfrak{a} \subseteq \mathfrak{N}_{L} \\
N<\mathfrak{\mathfrak { N }} \leq N^{\prime}}} \chi(\mathfrak{a}) e\left(h(\mathfrak{N a \mathfrak { a }})^{\delta}\right) \sum_{\substack{\mathfrak{b} \mathfrak{d}=\mathfrak{a} \\
\mathfrak{N} \mathfrak{b} \leq v, \mathfrak{N} \mathfrak{\mathfrak { c }} \leq u}} \mu_{L}(\mathfrak{b}) \Lambda_{L}(\mathfrak{c}) .
\end{aligned}
$$

2.1. Estimate of $S_{1}$. We first need an auxiliary result.

Lemma 11. Let $X, Y$ be positive integers and

$$
\alpha(m)=-\sum_{\substack{\mathfrak{c} \subseteq \mathfrak{O}_{L} \\ \mathfrak{N} \mathfrak{c}=m}} \chi(\mathfrak{c}) \Lambda_{L}(\mathfrak{c}), \quad \beta(n)=\sum_{\substack{\mathfrak{d} \subseteq \mathfrak{O}_{L} \\ \mathfrak{N} \mathfrak{l}=n}} \chi(\mathfrak{e}) \sum_{\substack{\mathfrak{b} \mathfrak{d}=\mathfrak{e} \\ \mathfrak{N} \mathfrak{b} \leq v}} \mu_{L}(\mathfrak{b}) .
$$

Then

$$
\sum_{X<m \leq 2 X}|\alpha(m)|^{2} \ll X(\log X)^{2 d_{L}-1}, \quad \sum_{Y<n \leq 2 Y}|\beta(n)|^{2} \ll Y(\log Y)^{4 d_{L}^{2}} .
$$

Proof. By the Cauchy-Schwarz inequality,

$$
\sum_{Y<n \leq 2 Y}|\beta(n)|^{2} \leq \sum_{Y \leq n \leq 2 Y}\left(\sum_{\substack{\mathfrak{e} \subseteq \mathfrak{O}_{L} \\ \mathfrak{N} \mathfrak{k}=n}} 1\right) \sum_{\substack{\mathfrak{e} \subseteq \mathfrak{O}_{L} \\ \mathfrak{N} \mathfrak{e}=n}}\left(\sum_{\substack{\mathfrak{b} \mathfrak{\mathfrak { N }}=\mathfrak{e} \\ \mathfrak{N} \mathfrak{b} \leq v}} \mu_{L}(\mathfrak{b})\right)^{2} \leq \sum_{Y \leq n \leq 2 Y} g(n)
$$


where $g(n)$ is the multiplicative function defined by

$$
g(n)=\left(\sum_{\substack{\mathfrak{e} \subseteq \mathfrak{O}_{L} \\ \mathfrak{N} \mathfrak{e}=n}} 1\right) \sum_{\substack{\mathfrak{e} \subseteq \mathfrak{O}_{L} \\ \mathfrak{N} \mathfrak{e}=n}} \tau^{2}(\mathfrak{e})
$$

and $\tau(\mathfrak{e})$ is the number of integral ideals of $L$ that divide $\mathfrak{e}$. Note that for any prime $p \geq 2$ we have $g(p) \leq 4 d_{L}^{2}$, while for $k>1$ the number of ideals $\mathfrak{e}$ with $\mathfrak{N e}=p^{k}$ is bounded by

$$
\left(\begin{array}{c}
d_{L}+k-1 \\
d_{L}-1
\end{array}\right)=e^{\sum_{m=1}^{k} \log \left(1+\frac{d_{L}-1}{m}\right)} \leq e^{\sum_{m=1}^{k} \frac{d_{L}-1}{m}} \leq(e k)^{d_{L}-1}
$$

and $\tau^{2}(\mathfrak{e}) \leq(k+1)^{2} \leq 4 k^{2}$. Thus, $g\left(p^{k}\right) \leq 4 e^{d_{L}-1} k^{d_{L}+1}$. It follows that

$$
\begin{aligned}
\log \left(1+\frac{g(p)}{p}+\frac{g\left(p^{2}\right)}{p^{2}}+\cdots\right) & =\log \left(1+\frac{g(p)}{p}\right)+O\left(1 / p^{2}\right) \\
& \leq \frac{4 d_{L}^{2}}{p}+O\left(1 / p^{2}\right)
\end{aligned}
$$

where the implied constant depends on $d_{L}$. Therefore,

$$
\begin{aligned}
\sum_{Y \leq n \leq 2 Y} g(n) & \leq 2 Y \sum_{Y \leq n \leq 2 Y} \frac{g(n)}{n} \leq 2 Y e^{\sum_{p \leq 2 Y} \log \left(1+\frac{g(p)}{p}+\frac{g\left(p^{2}\right)}{p^{2}}+\cdots\right)} \\
& \leq 2 Y e^{O(1)+4 d_{L}^{2} \sum_{p \leq 2 Y} 1 / p} \ll_{d_{L}} Y(\log Y)^{4 d_{L}^{2}}
\end{aligned}
$$

As for the other sum, we obtain

$$
\begin{aligned}
\sum_{X<m \leq 2 X}|\alpha(m)|^{2} & \leq \sum_{X \leq m \leq 2 X} \sum_{\substack{\mathfrak{c} \subseteq \mathcal{O}_{L} \\
\mathfrak{N} \mathfrak{c}=m}} 1 \cdot \sum_{\substack{\mathfrak{c} \subseteq \mathfrak{O}_{L} \\
\mathfrak{N} \mathfrak{c}=m}}\left(\Lambda_{L}(\mathfrak{c})\right)^{2} \\
& =\sum_{X \leq m \leq 2 X}(\Lambda(m))^{2}\left(\sum_{\substack{\mathfrak{c} \subseteq \mathfrak{O}_{L} \\
\mathfrak{N}=m}} 1\right)^{2} \ll d_{L}(\log X)^{2} \sum_{X \leq p^{k} \leq 2 X} k^{2\left(d_{L}-1\right)} \\
& \ll(\log X)^{2 d_{L}} \sum_{X \leq p^{k} \leq 2 X} 1 \ll X(\log X)^{2 d_{L}-1},
\end{aligned}
$$

as claimed.

We are now ready to estimate $S_{1}$. First, rewrite $S_{1}$ as

$$
\begin{aligned}
S_{1}= & \sum_{\substack{\mathfrak{c}, \mathfrak{e} \\
\mathfrak{N} \mathfrak{e}>v, \mathfrak{N} \mathfrak{c}>u \\
N \leq \mathfrak{N}(\mathfrak{c}) \leq N^{\prime}}} \chi(\mathfrak{e})\left(\sum_{\substack{\mathfrak{b} \mathfrak{b}=\mathfrak{e} \\
\mathfrak{N} \mathfrak{b} \leq v}} \mu_{L}(\mathfrak{b})\right) \chi(\mathfrak{c}) \Lambda_{L}(\mathfrak{c}) e\left(h(\mathfrak{N} \mathfrak{c})^{\delta}\right) \\
= & \sum_{\substack{n, m \\
n>v, m>u \\
N<n m \leq N^{\prime}}} \alpha(m) \beta(n) e\left(h(n m)^{\delta}\right)
\end{aligned}
$$


where $\alpha(m)$ and $\beta(n)$ are given by $(2.2)$. Let

$$
u=v=N^{\delta-1+\eta}
$$

and split the ranges of $m$ and $n$ into $\ll(\log N)^{2}$ subintervals of the form $[X, 2 X]$ and $[Y, 2 Y]$ such that $N / 4 \leq X Y \leq 2 N$ and $v<X, Y<N^{\prime} / v$. Summing over $h \leq H$ we conclude from Lemma 11 and [4, Lemma 4.13] with the exponent pair $(k, l)=(1 / 2,1 / 2)$ that the contribution of each subinterval is

$$
\begin{aligned}
\ll & \left(H^{7 / 6} N^{\delta / 6+5 / 6} \min \left(X^{-1 / 6}, Y^{-1 / 6}\right)+H N^{1 / 2} \max (X, Y)^{1 / 2}\right) \\
& \cdot(\log N)^{2 d_{L}^{2}+d_{L}+1 / 2} \\
\ll & \left(N^{2-1 / 12-\delta}+N^{5 / 2-3 \delta / 2-\eta / 2}\right) N^{8 \varepsilon / 6} .
\end{aligned}
$$

Finally, summing over $X$ and $Y$ we conclude that the estimate

$$
\sum_{h}\left|S_{1}\right| \ll N \exp \left(-D\left|\Delta_{K}\right|^{-1 / 2} \sqrt{\log N}\right)
$$

holds provided that

$$
1-\delta<\min \left(\frac{1}{12}, \frac{\eta}{3}\right)
$$

and $\varepsilon>0$ is sufficiently small, both of which we shall assume in what follows.

2.2. Estimate of $S_{3}$. We rewrite $S_{3}$ as $S_{4}+S_{5}$ where

$$
\begin{aligned}
S_{4} & =-\sum_{\substack{\mathfrak{e} \\
\mathfrak{N} \leq v}} \chi(\mathfrak{e})\left(\sum_{\substack{\mathfrak{b} \mathfrak{c}=\mathfrak{e} \\
\mathfrak{N} \mathfrak{b} \leq v, \mathfrak{N} \mathfrak{c} \leq u}} \mu_{L}(\mathfrak{b}) \Lambda_{L}(\mathfrak{c})\right) \sum_{\substack{\mathfrak{d} \\
N<\mathfrak{N}(\mathfrak{d}) \leq N^{\prime}}} \chi(\mathfrak{d}) e\left(h(\mathfrak{N}(\mathfrak{d} \mathfrak{e}))^{\delta}\right) \\
& \ll \log N \sum_{\substack{\mathfrak{e} \\
\mathfrak{N} \mathfrak{e} \leq v}} \sum_{\substack{\mathfrak{d} \\
N<\mathfrak{N}(\mathfrak{d}) \leq N^{\prime}}} \chi(\mathfrak{d}) e\left(h(\mathfrak{N}(\mathfrak{d} \mathfrak{e}))^{\delta}\right) \mid
\end{aligned}
$$

and

$$
\begin{aligned}
& S_{5}=-\sum_{\substack{\mathfrak{d}, \mathfrak{e} \\
v<\mathfrak{N} \leq v^{2} \\
N<\mathfrak{N}(\mathfrak{d}) \leq N^{\prime}}} \chi(\mathfrak{d}) \chi(\mathfrak{e})\left(\sum_{\substack{\mathfrak{h} \mathfrak{k}=\mathfrak{e} \\
\mathfrak{N} \mathfrak{b} \leq v, \mathfrak{N} \mathfrak{c} \leq u}} \mu_{L}(\mathfrak{b}) \Lambda_{L}(\mathfrak{c})\right) e\left(h(\mathfrak{N}(\mathfrak{d} \mathfrak{e}))^{\delta}\right) \\
& =\sum_{\substack{n, m \\
v<m \leq v^{2} \\
N<n m \leq N^{\prime}}} \alpha(m) \beta(n) e\left(h(n m)^{\delta}\right)
\end{aligned}
$$

with

$$
\alpha(m)=\sum_{\substack{\mathfrak{l} \\ \mathfrak{N} \mathfrak{e}=m}} \chi(\mathfrak{e})\left(\sum_{\substack{\mathfrak{b} \mathfrak{c}=\mathfrak{e} \\ \mathfrak{N} b \leq v, \mathfrak{N} \leq \leq u}} \mu_{L}(\mathfrak{b}) \Lambda_{L}(\mathfrak{c})\right), \quad \beta(n)=\sum_{\substack{\mathfrak{d} \\ \mathfrak{N} \mathfrak{d}=n}} \chi(\mathfrak{d}) .
$$

To estimate $S_{5}$ we split the ranges of $m$ and $n$ as we did for $S_{1}$ with the only difference that we now have $v<X \leq v^{2}$ and $N / v^{2}<Y<N^{\prime} / v$ 
in addition to $N / 4 \leq X Y \leq 2 N$. Furthermore, an analog of Lemma 11 can easily be established for the coefficients $\alpha(m)$ and $\beta(n)$; an explicit formulation will be omitted here. Using [4, Lemma 4.13] once again we see that the estimate

$$
\begin{aligned}
\sum_{h \leq H}\left|S_{5}\right| & \ll\left(N^{2-\delta-1 / 12}+N^{2-\delta} v^{-1 / 2}+N^{3 / 2-\delta} v\right) N^{2 \varepsilon} \\
& \ll N \exp \left(-D\left|\Delta_{K}\right|^{-1 / 2} \sqrt{\log N}\right)
\end{aligned}
$$

holds if we assume (2.4), that $\varepsilon>0$ is sufficiently small and that

$$
3 \eta \leq 1 \text {. }
$$

Finally, we note that $S_{4}$ can be estimated exactly the same way that $S_{2}$ will be handled in the next section. It does not impose any further restrictions on the range of $\delta$ than $S_{2}$ does, so we skip the details.

2.3. Estimate of $S_{2}$. We rewrite $S_{2}$ as

$$
S_{2}=\sum_{\substack{\mathfrak{d} \\ \mathfrak{N} \leq v}} \chi(\mathfrak{d}) \mu_{L}(\mathfrak{d}) \sum_{\substack{\mathfrak{c} \\ N / \mathfrak{N} \mathfrak{d}<\mathfrak{N} \leq N^{\prime} / \mathfrak{N o}}} \chi(\mathfrak{c}) e\left(h(\mathfrak{N} \mathfrak{c} \mathfrak{d})^{\delta}\right) \log \mathfrak{N} \mathfrak{c}
$$

and start with the estimation of

$$
S=\sum_{\substack{\mathfrak{c} \\ N / \mathfrak{N} \mathfrak{d}<\mathfrak{N} \mathfrak{c} \leq N^{\prime} / \mathfrak{N} \mathfrak{d}}} \chi(\mathfrak{c}) e\left(h(\mathfrak{N} \mathfrak{c} \mathfrak{d})^{\delta}\right) .
$$

Recall that $\chi$ is a ray class character of modulus $\mathfrak{f}$. Splitting $S$ into ray classes $\mathfrak{K}$ we obtain $S=\sum_{\mathfrak{K}} \chi(\mathfrak{K}) S_{\mathfrak{K}}$ where

$$
S_{\mathfrak{K}}=\sum_{\substack{\mathfrak{c} \in \mathfrak{K} \\ N / \mathfrak{N} \mathfrak{N}<\leq N^{\prime} / \mathfrak{N} \mathfrak{d}}} e\left(h(\mathfrak{N} \mathfrak{d} \mathfrak{d})^{\delta}\right) .
$$

Since there are only finitely many classes, it is enough to consider a fixed class $\mathfrak{K}$. Let $\mathfrak{b}$ be an integral ideal in the inverse class $\mathfrak{K}^{-1}$. Any integral ideal $\mathfrak{c} \in \mathfrak{K}$ is given by $\alpha \mathfrak{b}^{-1}$ for some $\alpha \in \mathfrak{b} \cap L_{\mathfrak{f}, 1}$, where

$$
L_{\mathfrak{f}, 1}:=\left\{x \in L^{*}: x \equiv 1 \bmod \mathfrak{f} \text {, and } x \text { is totally positive }\right\} .
$$

Thus, we have

$$
S_{\mathfrak{K}}=\sum_{\substack{\alpha \mathfrak{a} \\ \alpha \in \mathfrak{b} \cap L_{\mathfrak{f}, 1} \\ P^{d}<<\mathfrak{N}\left(\alpha \mathfrak{O}_{L}\right) \leq\left(P^{\prime}\right)^{d} L}} e\left(h(\mathfrak{N}(\alpha \mathfrak{a} \mathfrak{d}))^{\delta}\right)
$$

where $\mathfrak{a}=\mathfrak{b}^{-1}$,

$$
P=\left(\frac{N}{\mathfrak{N}(\mathfrak{a} \mathfrak{d})}\right)^{1 / d_{L}} \quad \text { and } \quad P^{\prime}=\left(\frac{N^{\prime}}{\mathfrak{N}(\mathfrak{a} \mathfrak{d})}\right)^{1 / d_{L}} .
$$


Since $\mathfrak{f}$ and $\mathfrak{b}$ are coprime ideals, we can find an $\alpha_{0} \in \mathfrak{b}$ such that $\alpha_{0} \equiv$ $1 \bmod \mathfrak{f}$. Hence, the condition that $\alpha \in \mathfrak{b} \cap L_{\mathfrak{f}, 1}$ is equivalent to the conditions that $\alpha \equiv \alpha_{0} \bmod \mathfrak{f b}$ and that $\alpha$ is totally positive.

Define a linear transformation $T$ from $L$ to the Minkowski space $L_{\mathbb{R}}:=$ $\left\{\left(z_{\tau}\right) \in L_{\mathbb{C}}: z_{\tau}=\bar{z}_{\bar{\tau}}\right\}$ by

$$
T \alpha=\left(\tau_{1} \alpha, \ldots, \tau_{d_{L}} \alpha\right)
$$

where $L_{\mathbb{C}}:=\prod_{\tau} \mathbb{C}$ and $\tau_{1}, \ldots, \tau_{d_{L}}$ are the embeddings of $L$ with the first $r_{1}$ embeddings being real and the first $r_{1}+r_{2}$ corresponding to the different archimedean valuations of $L$.

Note that $\alpha, \beta \in \mathfrak{b} \cap L_{\mathfrak{f}, 1}$ generate the same ideal if and only if they differ by a unit $u \in \mathfrak{O}_{L}^{*} \cap L_{\mathfrak{f}, 1}$. Since $\mathfrak{O}_{L}^{*} \cap L_{\mathfrak{f}, 1}$ is of finite index in $\mathfrak{O}_{L}^{*}$, its free part is of rank $r=r_{1}+r_{2}-1$. Let $\xi_{1}, \ldots, \xi_{r}$ be a system of fundamental units for $\mathfrak{O}_{L}^{*} \cap L_{\mathfrak{f}, 1}$, and $E$ the invertible $r \times r$ matrix whose rows are given by $\ell\left(T \xi_{1}\right), \ldots, \ell\left(T \xi_{r}\right)$ where $\ell: L_{\mathbb{C}}^{*}=\prod_{\tau} \mathbb{C}^{*} \rightarrow \mathbb{R}^{r}$ is defined by

$$
\ell\left(z_{1}, \ldots, z_{d_{L}}\right)=\left(\log \left|z_{1}\right|, \ldots, \log \left|z_{r}\right|\right) .
$$

If $L$ contains exactly $\omega$ roots of unity, then for any $t \in \mathbb{R}^{*}, \ell(T(t \alpha))=$ $\ell(T(t \beta))$ holds for exactly $\omega$ associates $\alpha$ of a given $\beta \in L^{*}$. Thus, in order to pick a representative $\alpha \in \mathfrak{b} \cap L_{\mathfrak{f}, 1}$ for the ideal $\alpha \mathfrak{a} \in \mathfrak{K}$ that is unique up to multiplication by roots of unity in $L$, we impose the condition that $\ell(T \alpha) E^{-1} \in[0,1)^{r}$. At this point, we define the set

$$
\Gamma_{0}:=\left\{\mathbf{z} \in L_{\mathbb{C}}^{*}: 1<\mathfrak{N} \mathbf{z} \leq N^{\prime} / N, \ell(\mathbf{z}) E^{-1} \in[0,1)^{r}, z_{1}, \ldots, z_{r_{1}}>0\right\}
$$

for the norm $\mathfrak{N} \mathbf{z}=\mathfrak{N}\left(z_{1}, \ldots, z_{d_{L}}\right):=\prod_{i} z_{i}$. Recalling the definition of $S_{\mathfrak{K}}$ above and noting that $\mathfrak{N} T \alpha=\mathfrak{N}_{L / \mathbb{Q}}(\alpha)$ for $\alpha \in L^{*}$, we see that

$$
\omega S_{\mathfrak{K}}=\sum_{\substack{\alpha \in \alpha_{0}+\mathfrak{f b} \\ T \alpha \in P \Gamma_{0}}} e\left(h(\mathfrak{N}(\alpha \mathfrak{a} \mathfrak{d}))^{\delta}\right) .
$$

Fix a $\mathbb{Z}$-basis $\left\{\alpha_{1}, \ldots, \alpha_{d_{L}}\right\}$ for the integral ideal $\mathfrak{f b}$ that satisfies 1.1 and let $M$ be the invertible matrix whose rows are given by $T \alpha_{1}, \ldots, T \alpha_{d_{L}}$. Since for $\alpha \in \alpha_{0}+\mathfrak{f b}, T \alpha$ can be written as $T \alpha_{0}+\mathbf{n} M$ for some unique $\mathbf{n} \in \mathbb{Z}^{d_{L}}$, we see that $\omega S_{\mathfrak{K}}=\sum_{\mathbf{n} \in \mathbb{Z}^{d} L} f(\mathbf{n})$, where $f: \mathbb{R}^{d_{L}} \rightarrow \mathbb{R}$ is given by

$$
f(\mathbf{x})= \begin{cases}e\left(D\left(\mathfrak{N}\left(\mathbf{x}_{0}+\mathbf{x} M\right)\right)^{\delta}\right) & \text { if } \mathbf{x}_{0}+\mathbf{x} M \in P \Gamma_{0}, \\ 0 & \text { otherwise, }\end{cases}
$$

$\mathbf{x}_{0}=T \alpha_{0}$, and $D=h(\mathfrak{N}(\mathfrak{a} \mathfrak{d}))^{\delta}$. Partitioning $\mathbb{R}^{d_{L}}$ into a disjoint union of translates $B$ of $[0, Y)^{d_{L}}$, where $Y \geq 1$ is an integer to be chosen later, we obtain

$$
\sum_{\mathbf{n} \in \mathbb{Z}^{d} L} f(\mathbf{n})=\sum_{B} \sum_{\mathbf{n} \in B \cap \mathbb{Z}^{d} L} f(\mathbf{n}) .
$$


Note that the condition $\ell(\mathbf{z}) E^{-1} \in[0,1)^{r}$ in the definition of $\Gamma_{0}$ above implies the existence of positive constants $c_{1}=c_{1}\left(d_{L}, \Delta_{L}\right)$ and $c_{2}=c_{2}\left(d_{L}, \Delta_{L}\right)$ such that for any $\alpha \in L^{*}$ with $T \alpha \in P \Gamma_{0}$ and any embedding $\tau$ of $L$, we have

$$
c_{1} P<|\tau \alpha|<c_{2} P .
$$

Let $\mathbf{R}$ be the region $\left\{\left(z_{1}, \ldots, z_{d_{L}}\right) \in L_{\mathbb{R}}: c_{1} P<\left|z_{i}\right|<c_{2} P\right\}$. Suppose that $f$ is not identically zero on $B \cap \mathbb{Z}^{d_{L}}$ for some $B$. If $\mathbf{x}_{0}+B M$ is partially contained in $\mathbf{R}$ then it must intersect the boundary of $\mathbf{R}$. Thus, we see that the contribution of such $B$ to the sum $\sum_{\mathbf{n}} f(\mathbf{n})$ is $O\left(Y P^{d_{L}-1}\right)$. For the rest of the boxes $B$ for which $f\left(B \cap \mathbb{Z}^{d_{L}}\right) \not \equiv 0$, we necessarily have $\mathbf{x}_{0}+B M \subseteq \mathbf{R}$. From now on, we assume that $B$ is such a box.

By the arguments in $\$ 2.7$, there exist constants $C_{1}=C_{1}\left(k, d_{L}, \Delta_{L}\right)$ and $C_{2}=C_{2}\left(k, d_{L}, \Delta_{L}\right)$ and a matrix $U \in \operatorname{SL}\left(d_{L}, \mathbb{Z}\right)$ such that for $N \geq C_{1}$, $1 \leq Y \leq C_{2} P$ and any $\mathbf{x}=\left(x_{1}, \ldots, x_{d_{L}}\right) \in B U^{-1}$, we have

$$
\left|\frac{\partial^{k}}{\partial x_{1}^{k}} g_{U}(\mathbf{x})\right| \asymp P^{\delta d_{L}-k} \quad \text { and } \quad \frac{\partial \lambda_{i}}{\partial x_{1}}(\mathbf{x}) \gg P^{-1}
$$

where $g_{U}$ is given by (2.13), $\lambda_{i}$ 's are determined by the condition $\ell\left(\mathbf{x}_{0}+\mathbf{x} U M\right)$ $=\left(\lambda_{1}(\mathbf{x}), \ldots, \lambda_{r}(\mathbf{x})\right) E$, and the implied constants depend on $k$ (only if relevant) and on $d_{L}$ and $\Delta_{L}$. After a change of variable we obtain

$$
\begin{aligned}
\sum_{\mathbf{n} \in B \cap \mathbb{Z}^{d} L} f(\mathbf{n}) & =\sum_{\mathbf{n} \in B U^{-1} \cap \mathbb{Z}^{d} L} f(\mathbf{n} U) \\
& =\sum_{\left(n_{2}, \ldots, n_{d_{L}}\right) \in \sum_{\mathbb{Z}^{d}-1}} \sum_{\substack{n_{1} \in \mathbb{Z} \\
\mathbf{n} \in B U^{-1} \cap \mathbb{Z}^{d} L}} f(\mathbf{n} U)
\end{aligned}
$$

where $\mathbf{n}=\left(n_{1}, \ldots, n_{d_{L}}\right)$. Since $f\left(B \cap \mathbb{Z}^{d_{L}}\right) \not \equiv 0$ there is at least one tuple $\left(n_{2}, \ldots, n_{d_{L}}\right)$ such that $f(\mathbf{n} U) \not \equiv 0$ for $n_{1} \in \mathbb{Z}$ and $\mathbf{n} \in B U^{-1} \cap \mathbb{Z}^{d_{L}}$. Fix such a tuple. It follows from (2.7) with $k=1$ that both $\lambda_{i}$ 's and the norm function are monotonic and thus there is an interval $I=I\left(n_{2}, \ldots, n_{d_{L}}\right)$ of length at most $O(Y)$ such that $f\left(x, n_{2}, \ldots, n_{d_{L}}\right) \neq 0$ for $x \in I$. We are now ready to estimate (2.8). We shall do so in what follows using different methods according to the size of the degree $d_{L}$ of the extension $L / \mathbb{Q}$.

2.4. Vinogradov's method-large degree. Assume that $d_{L} \geq 11$. It follows from (2.7) that there exist positive constants $C_{3}=C_{3}\left(d_{L}, \Delta_{L}\right)$ and $C_{4}=C_{4}\left(d_{L}, \Delta_{L}\right)$ such that

where

$$
\frac{1}{A_{0}} \leq\left|\frac{\partial^{d_{L}+1}}{\partial x_{1}^{d_{L}+1}}\left(D g_{U}(\mathbf{x})\right)\right| \leq \frac{C_{4}}{A_{0}}
$$

$$
A_{0}=\frac{P^{d_{L}(1-\delta)+1}}{C_{3} D}=\frac{N^{1-\delta+1 / d_{L}}}{C_{3} h(\mathfrak{N}(\mathfrak{a} \mathfrak{d}))^{1+1 / d_{L}}} .
$$


Using (2.1) and 2.3) we see that

$$
\frac{N^{1 / d_{L}-\varepsilon-\left(1+1 / d_{L}\right)(\eta+\delta-1)}}{C_{3}(\mathfrak{N}(\mathfrak{a}))^{1+1 / d_{L}}}<A_{0} \leq \frac{P^{d_{L}(1-\delta)+1}}{C_{3}(\mathfrak{N}(\mathfrak{a}))^{\delta}} .
$$

Therefore, assuming that $\eta<1 /\left(1+d_{L}\right)$ and $\varepsilon$ is sufficiently small it follows from Lemma 7 that for sufficiently large $N$, we have $A_{0}>1$. Set $\rho=$ $1 /\left(3 d_{L}^{2} \log \left(125 d_{L}\right)\right)$ and take

$$
Y=A_{0}^{1 /\left(\left(2+2 / d_{L}\right)(1-\rho)\right)} .
$$

Using equation (2.4), the upper bound for $A_{0}$ above and the inequality $\left(1+1 / d_{L}\right)(1-\rho)>1$, we deduce for sufficiently large $N$ that

$$
A_{0}^{1 /\left(2+2 / d_{L}\right)}<Y \leq \min \left(C_{2} P, A_{0}\right) .
$$

If the interval $I$ in $(2.8)$ satisfies

$$
A_{0}^{1 /\left(2+2 / d_{L}\right)} \ll|I|,
$$

we derive from (2.10) and [15, Theorem 2a, p. 109] that

$$
\sum_{\substack{n_{1} \in I \\ B U^{-1} \cap \mathbb{Z}^{d} L}} e\left(D g_{U}(\mathbf{n})\right) \ll|I|^{1-\rho} \ll Y^{1-\rho} .
$$

For smaller intervals $I$, trivially estimating the sum yields a contribution $\ll Y^{1-\rho}$ due to the choice of $Y$ in $(2.9)$. Since the number of tuples $\left(n_{2}, \ldots, n_{d_{L}}\right) \in \mathbb{Z}^{d_{L}-1}$ such that $\mathbf{n} \in B U^{-1} \cap \mathbb{Z}^{d_{L}}$ is $O\left(Y^{d_{L}-1}\right)$ we obtain

$$
\sum_{\mathbf{n} \in B \cap \mathbb{Z}^{d} L} f(\mathbf{n}) \ll Y^{d_{L}-\rho} .
$$

So, the contribution to the sum in (2.8) of those $B$ for which $f\left(B \cap \mathbb{Z}^{d_{L}}\right)$ $\not \equiv 0$ and $\mathbf{x}_{0}+B M \subseteq R$ is $\ll P^{d_{L}} Y^{-\rho}$, and this is already larger than the contribution from the rest of the boxes $B$.

Using (2.6) and partial summation and then summing over the ray classes $\mathfrak{K}$ we see that the sum

$$
\begin{aligned}
\sum_{\substack{\mathfrak{c} \\
N / \mathfrak{N} \mathfrak{d}<\mathfrak{N} \leq N^{\prime} / \mathfrak{N} \mathfrak{d}}} \chi(\mathfrak{c}) e\left(h(\mathfrak{N} \mathfrak{c} \mathfrak{d})^{\delta}\right) \log \mathfrak{N} \mathfrak{c} \\
\ll \frac{N}{\mathfrak{N} \mathfrak{d}}\left(\frac{N^{1-\delta+1 / d_{L}}}{h(\mathfrak{N} \mathfrak{d})^{1+1 / d_{L}}}\right)^{-\frac{\rho}{\left(2+2 / d_{L}\right)(1-\rho)}} \log N \\
=N^{1-\frac{\rho\left(1-\delta+1 / d_{L}\right)}{\left(2+2 / d_{L}\right)(1-\rho)}}(\mathfrak{N o})^{\frac{\rho}{2(1-\rho)}-1} h^{\frac{\rho}{\left(2+2 / d_{L}\right)(1-\rho)}} \log N .
\end{aligned}
$$

Finally, summing over ideals $\mathfrak{d}$ with $\mathfrak{N d} \leq v$ using the fact that $\sum_{\mathfrak{N} d \leq x} 1 \ll x$ (see for example [6, Ch. IV, Statement 2.15]) and then summing over $h$ with 
$h \leq H$ we deduce from 2.1 and 2.3 that

$$
\sum_{h \leq H}\left|S_{2}\right| \ll N^{1-\frac{\rho\left(1-\delta+1 / d_{L}\right)}{\left(2+2 / d_{L}\right)(1-\rho)}} v^{\frac{\rho}{2(1-\rho)}} H^{1+\frac{\rho}{\left(2+2 / d_{L}\right)(1-\rho)}} \log N \ll N^{1+q+2 \varepsilon}
$$

where

$$
q=\frac{1}{2(1-\rho)}\left(-\frac{\rho}{d_{L}+1}+(1-\delta)(2-3 \rho)+\rho \eta\right) .
$$

Thus, assuming (2.4) and choosing

$$
\frac{\eta}{3}=\frac{\rho}{2\left(d_{L}+1\right)}=\frac{1}{6\left(d_{L}+1\right) d_{L}^{2} \log \left(125 d_{L}\right)}
$$

we see that both (2.5) and the inequality $q<0$ hold. Hence for sufficiently large $N$ and sufficiently small $\varepsilon>0$,

$$
\sum_{h \leq H}\left|S_{2}\right| \ll N \exp \left(-D\left|\Delta_{K}\right|^{-1 / 2} \sqrt{\log N}\right)
$$

provided that $d_{L} \geq 11$.

2.5. Van der Corput's method—small degree. By [4, Theorem 2.8] and (2.7) we obtain

$$
\begin{aligned}
\sum_{\substack{n_{1} \\
\mathbf{n} \in B U^{-1} \cap \mathbb{Z}^{d} L}} e\left(D g_{U}(\mathbf{n})\right) \ll & Y \lambda^{1 /\left(2^{k+2}-2\right)}+Y^{1-1 / 2^{k+1}} \\
& +Y^{1-1 / 2^{k-1}+1 / 2^{2 k}} \lambda^{-1 / 2^{k+1}}
\end{aligned}
$$

where $\lambda:=D P^{d_{L} \delta-(k+2)}$. Note that this bound is no better than the trivial estimate unless $\lambda<1$. Therefore, we shall require that $\eta<1 /\left(d_{L}+1\right)$. With this assumption, we deduce that for $k \geq d_{L}-1$, for sufficiently large $N$ and sufficiently small $\varepsilon>0$, both of the inequalities $k+2>d_{L} \delta$ and $\lambda<1$ hold, since by (2.1), 2.3) and (2.4) we have

$$
\begin{aligned}
\lambda & =D P^{d_{L} \delta-(k+2)}=\frac{h(\mathfrak{N}(\mathfrak{a} \mathfrak{d}))^{\delta}}{(N /(\mathfrak{N a \mathfrak { d }}))^{\left(k+2-d_{L} \delta\right) / d_{L}}} \ll \frac{H N^{\delta}}{(N / v)^{(k+2) / d_{L}}} \\
& \ll N^{1+\frac{k+2}{d_{L}}(\eta+\delta-2)+\varepsilon} .
\end{aligned}
$$

We derive as before that the contribution from the boxes $B$ for which $f\left(B \cap \mathbb{Z}^{d_{L}}\right) \not \equiv 0$ and $\mathbf{x}_{0}+B M \subseteq R$ is

$$
\ll P^{d_{L}}\left(\lambda^{1 /\left(2^{k+2}-2\right)}+Y^{-1 / 2^{k+1}}+Y^{-1 / 2^{k-1}+1 / 2^{2 k}} \lambda^{-1 / 2^{k+1}}\right),
$$

while that from the rest of the boxes $B$ is $O\left(Y P^{d_{L}-1}\right)$. Combining these estimates yields the bound $S_{\mathfrak{K}} \ll P^{d_{L}}\left(\lambda^{1 /\left(2^{k+2}-2\right)}+G(Y)\right)$ where

$$
G(Y)=Y^{-1 / 2^{k+1}}+Y^{-1 / 2^{k-1}+1 / 2^{2 k}} \lambda^{-1 / 2^{k+1}}+Y P^{-1} .
$$


By [4, Lemma 2.4] it follows that for some $Y \in\left[1, C_{2} P\right]$,

$$
\begin{aligned}
G(Y) \ll & P^{-1 /\left(1+2^{k+1}\right)}+\left(P^{-1 / 2^{k-1}+1 / 2^{2 k}} \lambda^{-1 / 2^{k+1}}\right)^{1 /\left(1+1 / 2^{k-1}-1 / 2^{2 k}\right)} \\
& +P^{-1}+P^{-1 / 2^{k+1}}+\lambda^{-1 / 2^{k+1}} P^{-1 / 2^{k-1}+1 / 2^{2 k}} \\
\ll & P^{-1 /\left(1+2^{k+1}\right)}+\left(P^{-1 / 2^{k-1}+1 / 2^{2 k}} \lambda^{-1 / 2^{k+1}}\right)^{1 /\left(1+1 / 2^{k-1}-1 / 2^{2 k}\right)} .
\end{aligned}
$$

Note that in order to have $P^{-1 / 2^{k-1}+1 / 2^{2 k}} \lambda^{-1 / 2^{k+1}}<1$ one needs that $k<$ $d_{L}+2$, which can be seen using (2.1), (2.3), 2.4,$(2.6)$, and that $\eta<$ $1 /\left(d_{L}+1\right)$. Using equation $(2.6)$, the fact that $\lambda=D P^{d_{L^{\delta}-(k+2)}}$ and partial summation we derive that the sum

$$
(\log N)^{-1} \sum_{\substack{\mathfrak{c} \\ N / \mathfrak{N} \mathfrak{d}<\mathfrak{N} \mathfrak{c} \leq N^{\prime} / \mathfrak{N} \mathfrak{d}}} \chi(\mathfrak{c}) e\left(h(\mathfrak{N} \mathfrak{c} \mathfrak{d})^{\delta}\right) \log \mathfrak{N} \mathfrak{c}
$$

is

$$
\begin{aligned}
\ll & h^{1 /\left(2^{k+2}-2\right)} \mathfrak{N}(\mathfrak{d})^{\frac{k+2}{d_{L}\left(2^{k+2}-2\right)}-1} N^{1+\frac{d_{L} \delta-(k+2)}{d_{L}\left(2^{k+2}-2\right)}} \\
& +N^{1+\frac{1+2^{k-1}\left(k-2-d_{L} \delta\right)}{d_{L}\left(2^{2 k}+2^{k+1}-1\right)}}(\mathfrak{N} \mathfrak{d})^{-\frac{1+2^{k-1}(k-2)}{d_{L}\left(2^{2 k}+2^{k+1}-1\right)}-1} h^{-\frac{1}{2^{k+1}+4-2^{1-k}}} \\
& +(N / \mathfrak{N} \mathfrak{d})^{1-\frac{1}{d_{L}\left(1+2^{k+1}\right)}}
\end{aligned}
$$

Summing over ideals $\mathfrak{d}$ with $\mathfrak{N d} \leq v$, and then over $h \leq H$, we see that

$$
\begin{aligned}
(\log N)^{-1} & \sum_{h \leq H}\left|S_{2}\right| \\
\ll & H^{1+1 /\left(2^{k+2}-2\right)} v^{\frac{k+2}{d_{L}\left(2^{k+2}-2\right)}} N^{1+\frac{d_{L} \delta-(k+2)}{d_{L}\left(2^{k+2}-2\right)}}+H N^{1-\frac{1}{d_{L}\left(1+2^{k+1}\right)}} v^{\frac{1}{d_{L}\left(1+2^{k+1}\right)}} \\
& \quad+N^{1+\frac{1+2^{k-1}\left(k-2-d_{L} \delta\right)}{d_{L}\left(2^{2 k}+2^{k+1}-1\right)}} H^{1-\frac{1}{2^{k+1}+4^{1-k}}} \\
\ll & N^{1+q_{1}(k)+2 \varepsilon}+N^{1+q_{2}(k)+\varepsilon}+N^{1+q_{3}(k)+\varepsilon}
\end{aligned}
$$

where, assuming (2.5), it follows that the exponents $q_{i}(k)$ satisfy

$$
\begin{aligned}
q_{1}(k) & =(1-\delta)\left(1+\frac{1}{2^{k+2}-2}\right)+(\delta-1+\eta) \frac{k+2}{d_{L}\left(2^{k+2}-2\right)}+\frac{d_{L} \delta-(k+2)}{d_{L}\left(2^{k+2}-2\right)} \\
& <\frac{1}{d_{L}\left(2^{k+2}-2\right)}\left(\frac{\eta}{3}\left(d_{L}\left(2^{k+2}-2\right)+2 k+4\right)+d_{L}-k-2\right), \\
q_{2}(k) & =1-\delta-\frac{1}{d_{L}\left(1+2^{k+1}\right)}+(\delta-1+\eta) \frac{1}{d_{L}\left(1+2^{k+1}\right)} \\
& <\frac{1}{d_{L}\left(1+2^{k+1}\right)}\left(\frac{\eta}{3}\left(d_{L}\left(1+2^{k+1}\right)+2\right)-1\right),
\end{aligned}
$$


and

$$
\begin{aligned}
q_{3}(k) & =\frac{1+2^{k-1}\left(k-2-d_{L} \delta\right)}{d_{L}\left(2^{2 k}+2^{k+1}-1\right)}+(1-\delta)\left(1-\frac{1}{2^{k+1}+4-2^{1-k}}\right) \\
& <\frac{1+2^{k-1}\left(k-2-d_{L}\right)}{d_{L}\left(2^{2 k}+2^{k+1}-1\right)}+\frac{\eta}{3} .
\end{aligned}
$$

Thus, for sufficiently small $\varepsilon$, the estimate

$$
\sum_{h}\left|S_{2}\right| \ll N \exp \left(-D\left|\Delta_{K}\right|^{-1 / 2} \sqrt{\log N}\right)
$$

holds provided that for $1 \leq d_{L}-1 \leq k \leq d_{L}+1$,

$$
\begin{aligned}
\frac{\eta}{3}=\min \left(\frac{1}{3\left(d_{L}+1\right)+\varepsilon}\right. & , \frac{k+2-d_{L}}{d_{L}\left(2^{k+2}-2\right)+2 k+4}, \\
& \left.\frac{1}{d_{L}\left(1+2^{k+1}\right)+2}, \frac{2^{k-1}\left(d_{L}+2-k\right)-1}{d_{L}\left(2^{2 k}+2^{k+1}-1\right)}\right) .
\end{aligned}
$$

2.6. Conclusion of the proof of Theorem 2, Upon comparing (2.11) and $(2.12)$ we conclude that for $2 \leq d_{L}<11$, the maximum value for $\eta / 3$ (hence the widest range for $\delta$ ) is obtained via van der Corput's method when $k=d_{L}-1$ is substituted into the function

$$
\frac{k+2-d_{L}}{d_{L}\left(2^{k+2}-2\right)+2 k+4},
$$

while for $d_{L} \geq 11$ one needs to use Vinogradov's method, in which case we obtain

$$
\frac{\eta}{3}=\frac{1}{6\left(d_{L}+1\right) d_{L}^{2} \log \left(125 d_{L}\right)} .
$$

With the above choice of $\eta$, the claimed range for $c$ in Theorem 2 follows easily from 2.4.

REMARK 12. To estimate $S_{2}$, one may also use [14, Lemma 6.12] for $d_{L} \geq 7$, but the result is worse than what we have already obtained.

2.7. Derivative of the norm function. In this section we prove some auxiliary lemmas used in the estimate of $S_{2}$.

Lemma 13. Let $V \in \operatorname{GL}\left(d_{L}, \mathbb{R}\right), \mathbf{n} \in \mathbb{Z}^{d_{L}}$ and $\mathbf{x}, \mathbf{u} \in \mathbb{R}^{d_{L}}$. Set

$$
g_{V}(\mathbf{x})=\left|\mathfrak{N}\left(\mathbf{x}_{0}+\mathbf{x} V M\right)\right|^{\delta}, \quad \tilde{g}_{\mathbf{u}}(t)=\left|\mathfrak{N}\left(\mathbf{x}_{0}+\mathbf{n} M+t \mathbf{u} M\right)\right|^{\delta} .
$$

Then, for any $k \geq 1$, 


$$
\begin{aligned}
\left.\frac{\partial^{k} g_{V}}{\partial x_{1}^{k}}\right|_{\mathbf{x}=\mathbf{n} V^{-1}} & =\frac{d^{k}}{d t^{k}} \tilde{g}_{V_{1}}(0) \\
& =\sum_{\substack{i_{1}, \ldots, i_{k} \\
1 \leq i_{j} \leq d_{L}}} \ldots D_{i_{1}} \ldots D_{i_{k}} F\left(\mathbf{x}_{0}+\mathbf{n} M\right) v_{i_{1}} \cdots v_{i_{k}}
\end{aligned}
$$

where $F\left(z_{1}, \ldots, z_{d_{L}}\right)=\prod_{i=1}^{d_{L}} z_{i}^{\delta}, D_{i}=\partial / \partial z_{i}, v_{i}$ is the $i$ th component of the vector $V_{1} M$, and $V_{1}$ is the first row of $V$.

Proof. Use induction and the chain rule for derivatives.

Lemma 14. Given $\mathbf{a} \in \mathbf{R}$, there exists $\mathbf{v}=\mathbf{v}(\mathbf{a}) \in \mathbb{R}^{d_{L}}$ and a positive constant $\tilde{c}_{1}=\tilde{c}_{1}\left(k, d_{L}, \Delta_{L}\right)$ such that for any $k \geq 1$,

$$
\left|\frac{d^{k}}{d t^{k}} \tilde{g}(0)\right| \geq \tilde{c}_{1} P^{\delta d_{L}-k} \quad \text { where } \quad \tilde{g}(t)=|\mathfrak{N}(\mathbf{a}+t \mathbf{v} M)|^{\delta} .
$$

Proof. Assume first that $L$ has no real embeddings and that the first two coordinates in $L_{\mathbb{R}}$ correspond to conjugate embeddings. Write $\mathbf{a}=$ $\left(a_{1}, a_{2}, \ldots, a_{d_{L}}\right)$ and take $\mathbf{v}(\mathbf{a})=\left(\frac{a_{1}}{\left|a_{1}\right|}, \frac{a_{2}}{\left|a_{2}\right|}, 0, \ldots, 0\right) M^{-1}$. Note that $a_{1}=\bar{a}_{2}$ since $\mathbf{a} \in L_{\mathbb{R}}$. Using Lemma 13 with $V_{1}=\mathbf{v}$ and $\mathbf{x}_{0}+\mathbf{n} M=\mathbf{a}$ we see that

$$
\begin{aligned}
\frac{d^{k}}{d t^{k}} \tilde{g}(0) & =\sum_{\substack{i_{1}, \ldots, i_{k} \\
1 \leq i_{j} \leq d_{L}}} D_{i_{1}} \ldots D_{i_{k}} F(\mathbf{a}) v_{i_{1}} \cdots v_{i_{k}} \\
& =\sum_{j=0}^{k} \frac{k !}{j !(k-j) !} D_{1}^{j} D_{2}^{k-j} F(\mathbf{a})\left(\frac{a_{1}}{\left|a_{1}\right|}\right)^{j}\left(\frac{a_{2}}{\left|a_{2}\right|}\right)^{k-j} \\
& =\frac{k ! F(\mathbf{a})}{\left|a_{1}\right|^{k}} \sum_{j}\left(\begin{array}{l}
\delta \\
j
\end{array}\right)\left(\begin{array}{c}
\delta \\
k-j
\end{array}\right)=\frac{k ! F(\mathbf{a})}{\left|a_{1}\right|^{k}}\left(\begin{array}{l}
2 \\
\delta
\end{array}\right) k
\end{aligned}
$$

where $\left(\begin{array}{l}\delta \\ j\end{array}\right)$ is the coefficient of $x^{j}$ in the Taylor series expansion of $(1+x)^{\delta}$ and the last equality follows by writing $(1+x)^{2 \delta}=(1+x)^{\delta}(1+x)^{\delta}$ in two ways as series and comparing the coefficients of $x^{k}$. Since $\mathbf{a} \in \mathbf{R}$, we have $c_{1} P<\left|a_{i}\right|<c_{2} P$ for each $i$. We thus obtain

$$
\left|\frac{d^{k}}{d t^{k}} \tilde{g}(0)\right| \geq c_{1}^{\delta d_{L}} c_{2}^{-k} P^{\delta d_{L}-k} k !\left|\left(\begin{array}{c}
2 \delta \\
k
\end{array}\right)\right| .
$$

If $L$ has at least one real embedding, take $\mathbf{v}=(1,0, \ldots, 0) M^{-1}$. In this case, Lemma 13 gives

$$
\left|\frac{d^{k}}{d t^{k}} \tilde{g}(0)\right|=\left|\delta(\delta-1) \cdots(\delta-k+1) F(\mathbf{a}) a_{1}^{-k}\right| \geq c_{1}^{\delta d_{L}} c_{2}^{-k} P^{\delta d_{L}-k} k !\left|\left(\begin{array}{l}
\delta \\
k
\end{array}\right)\right| .
$$

Since $\delta \in(1 / 2,1)$ and is fixed, we obtain the claimed lower bound. 
Lemma 15. Given $\mathbf{a}=\mathbf{x}_{0}+\mathbf{n} M \in \mathbf{R}$ where $\mathbf{n} \in \mathbb{Z}^{d_{L}}$, there exists a matrix $U \in \mathrm{SL}\left(d_{L}, \mathbb{Z}\right)$ such that for any $k \geq 1$,

$$
\frac{\partial^{k} g_{U}\left(\mathbf{n} U^{-1}\right)}{\partial x_{1}^{k}} \gg P^{\delta d_{L}-k}, \quad \frac{\partial \lambda_{i}\left(\mathbf{n} U^{-1}\right)}{\partial x_{1}} \gg P^{-1} \quad(i=1, \ldots, r)
$$

where $g_{U}$ is given by (2.13) and the implied constants depend on $d_{L}$ and $\Delta_{L}$, with the first one also depending on $k$.

Proof. Using Lemma 14 we find a vector $\tilde{\mathbf{v}}=\left(\tilde{v}_{1}, \ldots, \tilde{v}_{d_{L}}\right) \in \mathbb{R}^{d_{L}}$. Set $\mathbf{v}=\tilde{\mathbf{v}} M=\left(v_{1}, \ldots, v_{d_{L}}\right)$. Suppose that for some $Q>0$, there exists $\tilde{\mathbf{u}}=$ $\left(\tilde{u}_{1}, \ldots, \tilde{u}_{d_{L}}\right) \in \mathbb{Z}^{d_{L}}$ such that $\left|\tilde{u}_{i}-Q \tilde{v}_{i}\right|<1$. Set $\mathbf{u}=\tilde{\mathbf{u}} M$ and $\mathbf{w}=\mathbf{u}-Q \mathbf{v}=$ $\left(w_{1}, \ldots, w_{d_{L}}\right)$. By Lemma 13 we see that

$$
\begin{aligned}
\frac{d^{k}}{d t^{k}} \tilde{g}_{\tilde{\mathbf{u}}}(0) & =\sum_{\substack{i_{1}, \ldots, i_{k} \\
1 \leq i_{j} \leq d_{L}}} D_{i_{1}} \ldots D_{i_{k}} F(\mathbf{a}) \prod_{l=1}^{k}\left(Q v_{i_{l}}+w_{i_{l}}\right) \\
& =\sum_{\substack{i_{1}, \ldots, i_{k} \\
1 \leq i_{j} \leq d_{L}}} D_{i_{1}} \ldots D_{i_{k}} F(\mathbf{a})\left(Q^{k} v_{i_{1}} \cdots v_{i_{k}}+\sum_{l=1}^{k} Q^{k-l} A_{l}(\mathbf{v}, \mathbf{w})\right) \\
& =Q^{k} \frac{d^{k}}{d t^{k}} \tilde{g}_{\tilde{\mathbf{v}}}(0)+\sum_{l=1}^{k} Q^{k-l} \sum_{\substack{i_{1}, \ldots, i_{k} \\
1 \leq i_{j} \leq d_{L}}} D_{i_{1}} \ldots D_{i_{k}} F(\mathbf{a}) A_{l}(\mathbf{v}, \mathbf{w}) .
\end{aligned}
$$

Write $D_{i_{1}} \ldots D_{i_{k}} F(\mathbf{a})$ by grouping the same indices as $D_{j_{1}}^{l_{1}} \ldots D_{j_{r}}^{l_{r}} F(\mathbf{a})$ with $j_{i}$ 's distinct and $\sum_{i} l_{i}=k$. Since $\mathbf{a} \in \mathbf{R}$, we have $c_{1} P<\left|a_{i}\right|<c_{2} P$ for each $i$. Thus,

$$
\begin{aligned}
\left|D_{j_{1}}^{l_{1}} \ldots D_{j_{r}}^{l_{r}} F(\mathbf{a})\right| & =|F(\mathbf{a})| \prod_{i} \frac{\left|\delta(\delta-1) \cdots\left(\delta-l_{i}+1\right)\right|}{\left|a_{i}\right|^{l_{i}}} \\
& \leq\left(c_{2} P\right)^{\delta d_{L}} \prod_{i} \frac{\left|\delta(\delta-1) \cdots\left(\delta-l_{i}+1\right)\right|}{\left(c_{1} P\right)^{l_{i}}} \leq c_{3} P^{\delta d_{L}-k}
\end{aligned}
$$

for some constant $c_{3}=c_{3}\left(k, d_{L}, \Delta_{L}\right)>0$. Owing to the way $\tilde{\mathbf{v}}$ is constructed in Lemma 14. $\left|v_{i}\right| \leq 1$ for each $i$. Furthermore, each $w_{i}$ is bounded only in terms of $d_{L}$ and $\Delta_{L}$. Therefore, there exists a constant $c_{4}=c_{4}\left(k, d_{L}, \Delta_{L}\right)$ such that $\left|A_{l}(\mathbf{v}, \mathbf{w})\right| \leq c_{4}$. We thus conclude from Lemma 14 that

$$
\begin{aligned}
\left|\frac{d^{k}}{d t^{k}} \tilde{g}_{\tilde{\mathbf{u}}}(0)\right| & \geq Q^{k}\left|\frac{d^{k}}{d t^{k}} \tilde{g}_{\tilde{\mathbf{v}}}(0)\right|-\sum_{l=1}^{k} Q^{k-l}\left|\sum_{\substack{i_{1}, \ldots, i_{k} \\
1 \leq i_{j} \leq d_{L}}} D_{i_{1}} \ldots D_{i_{k}} F(\mathbf{a}) A_{l}(\mathbf{v}, \mathbf{w})\right| \\
& \geq P^{\delta d_{L}-k}\left(\tilde{c}_{1} Q^{k}-C_{k-1} Q^{k-1}-\cdots-C_{1} Q-C_{0}\right)
\end{aligned}
$$

for some constants $C_{i}=C_{i}\left(k, d_{L}, \Delta_{L}\right)>0$. 
Next, let $G_{U}(\mathbf{x})=\ell\left(\mathbf{x}_{0}+\mathbf{x} U M\right) E^{-1}$. Note that $\lambda_{i}(\mathbf{x})$ is the $i$ th coordinate of this function. Writing $\mathbf{a}=\left(a_{1}, \ldots, a_{d_{L}}\right)$ and $\mathbf{u}=\left(u_{1}, \ldots, u_{d_{L}}\right)$ we get

$$
\left.\frac{\partial G_{U}(\mathbf{x})}{\partial x_{1}}\right|_{\mathbf{x}=\mathbf{n} U^{-1}}=\left(\operatorname{Re}\left(\frac{u_{1}}{a_{1}}\right), \ldots, \operatorname{Re}\left(\frac{u_{r}}{a_{r}}\right)\right) E^{-1}
$$

where $\operatorname{Re}(z)$ denotes the real part of $z$. Recalling that $u_{i}=Q v_{i}+w_{i}$ we conclude as before that

$$
\left|\frac{\partial \lambda_{i}\left(\mathbf{n} U^{-1}\right)}{\partial x_{1}}\right| \geq P^{-1}\left(\tilde{C}_{1} Q-\tilde{C}_{0}\right)
$$

for some positive constants $\tilde{C}_{1}$ and $\tilde{C}_{0}$ that depend only on $d_{L}$ and $\Delta_{L}$.

It follows that there exists a constant $Q_{0}=Q_{0}\left(k, d_{L}, \Delta_{L}\right)>0$ such that both polynomials in $Q$ above are positive for $Q>Q_{0}$. If all the components of $\tilde{\mathbf{v}}$ are equal we fix some $Q>Q_{0}$ and let $\tilde{u}_{1}=\left\lceil Q \tilde{v}_{1}\right\rceil$ and $\tilde{u}_{i}=\left\lfloor Q \tilde{v}_{1}\right\rfloor$ (if any $\tilde{u}_{i}$ turns out to be zero, we can instead choose all $\left.\tilde{u}_{i}=1\right)$. Otherwise, find the first index $i_{0}$ such that $\left|\tilde{v}_{i_{0}}\right|=\max _{i}\left|\tilde{v}_{i}\right|$ and choose $Q=(p-1 / 2) /\left|\tilde{v}_{i_{0}}\right|$ where $p$ is the smallest prime $>Q_{0}\left|\tilde{v}_{i_{0}}\right|$. Choose $\tilde{u}_{i_{0}}= \pm p$ depending on the sign of $\tilde{v}_{i_{0}}$, and the rest of the $\tilde{u}_{j}$ 's as either the ceiling or the floor of $Q \tilde{v}_{j}$ so that $0<\left|\tilde{u}_{j}\right|<\left|\tilde{u}_{i_{0}}\right|=p$ for $j \neq i_{0}$. In either case, we can find a vector $\tilde{\mathbf{u}} \in \mathbb{Z}^{d_{L}}$ that satisfies $\left|\tilde{u}_{i}-Q \tilde{v}_{i}\right|<1$ and $\operatorname{gcd}\left(\tilde{u}_{1}, \ldots, \tilde{u}_{d_{L}}\right)=1$. It follows from [11, Corollary II.1] that $\tilde{\mathbf{u}}$ can then be completed to a matrix $U \in \operatorname{SL}\left(d_{L}, \mathbb{Z}\right)$ with $\tilde{\mathbf{u}}$ as the first row. Thus, the claimed lower bound follows by noting that

$$
\frac{\partial^{k} g_{U}\left(\mathbf{n} U^{-1}\right)}{\partial x_{1}^{k}}=\frac{d^{k}}{d t^{k}} \tilde{g}_{\tilde{\mathbf{u}}}(0) \gg P^{\delta d_{L}-k}
$$

Suppose now that $\mathbf{x}_{0}+\mathbf{n} M \in P \Gamma_{0}$ for some $\mathbf{n} \in B \cap \mathbb{Z}^{d_{L}}$. It follows from Lemma 15 with $\mathbf{a}=\mathbf{x}_{0}+\mathbf{n} M$ that there exists a matrix $U$ such that the inequality

$$
\left|\frac{\partial^{k}}{\partial x_{1}^{k}} g_{U}(\mathbf{x})\right| \geq c_{3} P^{\delta d_{L}-k}
$$

holds for some positive constant $c_{3}=c_{3}\left(k, d_{L}, \Delta_{L}\right)$ where $\mathbf{x}=\mathbf{n} U^{-1}$. If $\mathbf{x}^{\prime}$ is any other point in $B U^{-1}$ it follows from the Mean Value Theorem for integrals, Lemma 13 and the inclusion $\mathbf{x}_{0}+B M \subseteq \mathbf{R}$ that

$$
\frac{\partial^{k}}{\partial x_{1}^{k}} g_{U}(\mathbf{x})-\frac{\partial^{k}}{\partial x_{1}^{k}} g_{U}\left(\mathbf{x}^{\prime}\right)=\int_{0}^{1} \frac{d}{d t}\left(\frac{\partial^{k}}{\partial x_{1}^{k}} g_{U}\left(t \mathbf{x}+(1-t) \mathbf{x}^{\prime}\right)\right) d t \ll Y P^{\delta d_{L}-k-1}
$$

where the implied constant, say $c_{4}$, depends on $k, d_{L}$, and $\Delta_{L}$. In particular, it does not depend on the choice of $\mathbf{x}^{\prime} \in B U^{-1}$. Thus, for any point $\mathbf{x}^{\prime} \in B U^{-1}$, the lower bound

$$
\left|\frac{\partial^{k}}{\partial x_{1}^{k}} g_{U}\left(\mathbf{x}^{\prime}\right)\right| \geq \frac{c_{3}}{2} P^{\delta d_{L}-k}
$$


holds provided that $1 \leq Y \leq c_{3} P /\left(2 c_{4}\right)$. This condition imposes a further restriction on $N$, namely $N^{2-\delta-\eta} \geq \mathfrak{N a}\left(2 c_{4} / c_{3}\right)^{d_{L}}$. Assuming $\eta<1 / d_{L}$ and that $\mathfrak{N a}$ is bounded (which follows from Lemma 7), we deduce that for sufficiently large $N$, and all $\mathbf{x}^{\prime} \in B U^{-1}$,

$$
\frac{\partial^{k}}{\partial x_{1}^{k}} g_{U}\left(\mathbf{x}^{\prime}\right) \asymp P^{\delta d_{L}-k}
$$

where the implied constants depend only on $k, d_{L}$ and $\Delta_{L}$ provided $1 \leq$ $Y \ll P$. Using the same argument we can also show that $\lambda_{i}$ 's are monotonic in the first variable on $B U^{-1}$.

3. Proof of Theorem 1. By the definition of the conductor (cf. [10, Ch. VI, (6.3) and (6.4)]), $K^{\dagger} / K$ is the smallest ray class field containing the abelian extension $K / \mathbb{Q}$. Furthermore, every ray class field over $\mathbb{Q}$ corresponds to a cyclotomic extension. In particular, it follows from [10, Ch. VI, Proposition (6.7)] that there is an integer $q$ such that $\mathfrak{f}=(q)$ and $K^{\mathfrak{f}}$ is the $q$ th cyclotomic extension of $\mathbb{Q}$.

Fix $\sigma_{0} \in G$ and set $A_{0}=\left\{\sigma \in \operatorname{Gal}(L / \mathbb{Q}): \sigma_{\left.\right|_{K}}=\sigma_{0}\right\}$ where $\sigma_{\left.\right|_{K}}$ is the restriction of $\sigma$ to $K$. Then it follows from [6, Ch. III, Property 2.4] that the set $\pi\left(K,\left\{\sigma_{0}\right\}\right)$ is the disjoint union of the sets $\pi(L,\{\sigma\})$ for $\sigma \in A_{0}$. Therefore,

$$
\pi_{c}\left(K,\left\{\sigma_{0}\right\}, x\right)=\sum_{\sigma \in A_{0}} \pi_{c}(L,\{\sigma\}, x) .
$$

Since each $\sigma \in A_{0}$ corresponds to some $a_{\sigma} \in(\mathbb{Z} / q \mathbb{Z})^{*}$ we have $\pi_{c}(L,\{\sigma\}, x)$ $=\pi_{c}\left(x ; q, a_{\sigma}\right)$, where the latter counts the Piatetski-Shapiro primes not exceeding $x$ that are congruent to $a_{\sigma}$ modulo $q$.

By [9, Corollary 11.21] there exists an absolute constant $D>0$ and a constant $x_{0}(\mathfrak{f})$ such that for $x \geq x_{0}(\mathfrak{f})$ we have

$$
\sum_{\substack{p \leq x \\ p \equiv a_{\sigma} \bmod q}}\left((p+1)^{\delta}-p^{\delta}\right)=\frac{\delta}{\varphi(q)} \operatorname{li}\left(x^{\delta}\right)+O\left(x^{\delta} \exp (-D \sqrt{\log x})\right)
$$

where the implied constant is absolute. Furthermore, as in the proof of Theorem 2, choosing $H=N^{1-\delta+\varepsilon}$ we derive that

$$
\begin{aligned}
& \pi_{c}\left(x ; q, a_{\sigma}\right)-\sum_{\substack{p \leq x \\
p \equiv a_{\sigma} \bmod q}}\left((p+1)^{\delta}-p^{\delta}\right) \\
& \ll \sum_{\substack{1 \leq N<x \\
N=2^{k}}} N^{\delta-1} \sum_{h \leq H} \max _{N^{\prime} \in\left(N, N_{1}\right]}\left|\sum_{\substack{N<n \leq N^{\prime} \\
n \equiv a_{\sigma} \bmod q}} e\left(h n^{\delta}\right) \Lambda(n)\right|+x^{\delta} \exp (-D \sqrt{\log x})
\end{aligned}
$$

where $D$ is the same constant as above. Thus, to finish the proof it suffices 
to show that for any $N^{\prime} \in\left(N, N_{1}\right]$,

$$
\sum_{h \leq H}\left|\sum_{\substack{N<n \leq N^{\prime} \\ n \equiv a_{\sigma} \bmod q}} e\left(h n^{\delta}\right) \Lambda(n)\right| \ll N \exp (-D \sqrt{\log N}) .
$$

Applying Vaughan's identity (see, e.g., [5, Proposition 13.4]) and assuming that $v=u<N$ we obtain

$$
\sum_{\substack{N<n \leq N^{\prime} \\ n \equiv a_{\sigma} \bmod q}} e\left(h n^{\delta}\right) \Lambda(n)=S_{1}+S_{2}+S_{3}
$$

where

$$
\begin{aligned}
S_{1} & =-\sum_{\substack{N<n \leq N^{\prime} \\
n \equiv a_{\sigma} \bmod q}} e\left(h n^{\delta}\right) \sum_{\substack{n=c d \\
c, d>v}} \Lambda(c) \sum_{\substack{d=a b \\
b \leq v}} \mu(b), \\
S_{2} & =\sum_{\substack{N<n \leq N^{\prime} \\
n \equiv a_{\sigma} \bmod q}} e\left(h n^{\delta}\right) \sum_{\substack{n=a b \\
b \leq v}} \mu(b) \log a, \\
S_{3} & =-\sum_{\substack{N<n \leq N^{\prime} \\
n \equiv a_{\sigma} \bmod q}} e\left(h n^{\delta}\right) \sum_{\substack{n=a b c \\
b, c \leq v}} \mu(b) \Lambda(c) .
\end{aligned}
$$

Using Dirichlet characters $\chi$ modulo $q$ we obtain

$$
S_{1}=-\frac{1}{\varphi(q)} \sum_{\chi \bmod q} \overline{\chi\left(a_{\sigma}\right)} \sum_{\substack{N<c d \leq N^{\prime} \\ c, d>v}} \chi(d)\left(\sum_{\substack{d=a b \\ b \leq v}} \mu(b)\right) \chi(c) \Lambda(c) e\left(h(c d)^{\delta}\right),
$$

where $\varphi$ is Euler's totient function. By [4, Lemma 4.13] we conclude as in the non-abelian case that

$$
N^{-4 \varepsilon / 3} \sum_{h}\left|S_{1}\right| \ll N^{2-1 / 12-\delta}+N^{2-\delta} v^{-1 / 2} .
$$

For $S_{2}$, we use additive characters modulo $q$ to obtain

$$
S_{2}=\frac{1}{q} \sum_{k=0}^{q-1} e\left(-k a_{\sigma} / q\right) \sum_{b \leq v} \mu(b) \sum_{\substack{a \\ N / b<a \leq N^{\prime} / b}} e(f(a)) \log a
$$

where $f(x)=h b^{\delta} x^{\delta}+k b x / q$. Since $\left|f^{\prime \prime}(x)\right| \asymp h b^{2} N^{\delta-2}$ for $N / b<x \leq N^{\prime} / b$ we conclude from [4, Theorem 2.2] that

$$
\sum_{\substack{a \\ N / b<a \leq N^{\prime} / b}} e\left(h(a b)^{\delta}+k a b / q\right) \ll N^{\delta / 2} h^{1 / 2}+h^{-1 / 2} b^{-1} N^{1-\delta / 2} .
$$

Using partial summation and then summing over $b \leq v$ followed by $h \leq H$, 
we obtain

$$
\sum_{h}\left|S_{2}\right| \ll\left(N^{\delta / 2} H^{3 / 2} v+H^{1 / 2} N^{1-\delta / 2}\right) \log ^{2} N \ll N^{3 / 2-\delta+2 \varepsilon} v
$$

Write $S_{3}$ as $-S_{4}-S_{5}$, where

$$
\begin{aligned}
S_{4} & =\sum_{d \leq v} \sum_{d=b c} \mu(b) \Lambda(c) \sum_{\substack{N / d<a \leq N^{\prime} / d \\
a d \equiv a_{\sigma} \bmod q}} e\left(h(a d)^{\delta}\right) \\
& \ll \log N \sum_{d \leq v}\left|\sum_{\substack{N / d<a \leq N^{\prime} / d \\
a d \equiv a_{\sigma} \bmod q}} e\left(h(a d)^{\delta}\right)\right|,
\end{aligned}
$$

and

$$
S_{5}=\sum_{\substack{N<a d \leq N^{\prime} \\ a d \equiv a_{\sigma} \bmod q \\ u<d \leq v^{2}}} e\left(h(a d)^{\delta}\right) \sum_{\substack{d=b c \\ b, c \leq v}} \mu(b) \Lambda(c) .
$$

Applying [4, Lemma 4.13] once again we conclude as we did for $S_{1}$ above that

$$
N^{-4 \varepsilon / 3} \sum_{h}\left|S_{5}\right| \ll N^{2-\delta-1 / 12}+N^{2-\delta} v^{-1 / 2}+N^{3 / 2-\delta} v .
$$

Finally, we note that $S_{4}$ can be handled exactly the same way as $S_{2}$. Choosing $v=N^{\delta-1 / 2-3 \varepsilon}$ with a sufficiently small $\varepsilon$ and combining all the estimates obtained above we see that

$$
\sum_{h \leq H}\left|\sum_{\substack{N<n \leq N^{\prime} \\ n \equiv a_{\sigma} \bmod q}} e\left(h n^{\delta}\right) \Lambda(n)\right| \ll N \exp (-D \sqrt{\log N}),
$$

as desired, provided that $c \in(1,12 / 11)$.

The proof of Theorem 1 is thus completed by noting that the number of elements in $A_{0}$ equals $|\operatorname{Gal}(L / K)|=\varphi(q)\left|\Delta_{K}\right|^{-1}$.

Acknowledgements. We are much obliged to Professor Roger HeathBrown for his invaluable suggestions and comments on this work. We also thank the referee for carefully reading the manuscript and making helpful suggestions. This work was partially supported by TÜBITAK grant $112 \mathrm{~T} 180$.

\section{References}

[1] R. C. Baker, W. D. Banks, J. Brüdern, I. E. Shparlinski and A. J. Weingartner, Piatetski-Shapiro sequences, Acta Arith. 157 (2013), 37-68.

[2] K. M. Bartz, On a theorem of A. V. Sokolovskiน, Acta Arith. 34 (1977/78), 113-126. 
[3] D. A. Cox, Primes of the Form $x^{2}+n y^{2}$. Fermat, Class Field Theory and Complex Multiplication, Wiley, New York, 1989.

[4] S. W. Graham and G. Kolesnik, Van der Corput's Method of Exponential Sums, London Math. Soc. Lecture Note Ser. 126, Cambridge Univ. Press, Cambridge, 1991.

[5] H. Iwaniec and E. Kowalski, Analytic Number Theory, Amer. Math. Soc. Colloq. Publ. 53, Amer. Math. Soc., Providence, RI, 2004.

[6] G. J. Janusz, Algebraic Number Fields, Pure Appl. Math. 55, Academic Press, New York, 1973.

[7] J. C. Lagarias and A. M. Odlyzko, Effective versions of the Chebotarev density theorem, in: Algebraic Number Fields: $L$-functions and Galois Properties (Durham, 1975), Academic Press, London, 1977, 409-464.

[8] D. Leitmann und D. Wolke, Primzahlen der Gestalt $\left[n^{\Gamma}\right]$ in arithmetischen Progressionen, Arch. Math. (Basel) 25 (1974), 492-494.

[9] H. L. Montgomery and R. C. Vaughan, Multiplicative Number Theory. I. Classical Theory, Cambridge Stud. Adv. Math. 97, Cambridge Univ. Press, Cambridge, 2007.

[10] J. Neukirch, Algebraic Number Theory, Grundlehren Math. Wiss. 322, Springer, Berlin, 1999.

[11] M. Newman, Integral Matrices, Pure Appl. Math. 45, Academic Press, New York, 1972.

[12] I. I. Piatetski-Shapiro, On the distribution of prime numbers in sequences of the form $[f(n)]$, Mat. Sb. (N.S.) 33 (75) (1953), 559-566 (in Russian).

[13] J. Rivat et P. Sargos, Nombres premiers de la forme $\left\lfloor n^{c}\right\rfloor$, Canad. J. Math. 53 (2001), 414-433.

[14] E. C. Titchmarsh, The Theory of the Riemann Zeta-Function, 2nd ed., Clarendon Press, New York, 1986.

[15] I. M. Vinogradov, The Method of Trigonometrical Sums in the Theory of Numbers, Dover Publ., Mineola, NY, 2004.

Yıldırım Akbal, Ahmet Muhtar Güloğlu

Department of Mathematics

Bilkent University

06800 Bilkent, Ankara, Turkey

E-mail: yildirim.akbal@bilkent.edu.tr guloglua@fen.bilkent.edu.tr

Received on 12.12.2013

and in revised form on 19.1.2015 
